Aus der Klinik für Psychiatrie und Psychotherapie,

Universitätsklinikum des Saarlandes, Homburg/Saar

Direktor: Prof. Dr. med. M. Riemenschneider

\title{
Psychiatrische Versorgung in der Notaufnahme
}

Dissertation zur Erlangung des Grades eines Doktors der Medizin der Medizinischen Fakultät

der UNIVERSITÄT DES SAARLANDES

2011

vorgelegt von: Erik Rainer Puffer

geboren am: 26.04.1966 in Viersen 
Marlene Kentgens in Liebe und Dankbarkeit gewidmet 


\section{INHALTSVERZEICHNIS}

1. ZUSAMMENFASSUNG 5

$1.1 \quad$ Zusammenfassung 5

$\begin{array}{llr}1.2 & \text { Summary } & 6\end{array}$

$\begin{array}{lll}\text { 2. } & \text { EINLEITUNG } & 8\end{array}$

3. MATERIAL UND METHODEN 10

$\begin{array}{lll}3.1 & \text { Untersuchungsdesign } & 10\end{array}$

$\begin{array}{lll}3.2 & \text { Untersuchungsart und Zeitraum } & 10\end{array}$

$\begin{array}{lll}3.3 & \text { Fragebogen und Datenerfassung } & 11\end{array}$

$\begin{array}{lll}3.4 & \text { Kodierung und Datenerhebung } & 15\end{array}$

$\begin{array}{lll}3.5 & \text { Statistische Auswertung } & 15\end{array}$

$\begin{array}{ll}\text { 4. ERGEBNISSE } & 16\end{array}$

$\begin{array}{lll}\text { 4.1 Demographische Daten und Compliance } & 16\end{array}$

$\begin{array}{lll}4.2 & \text { Art der Krankenhäuser und der Notaufnahmen } & 16\end{array}$

4.3 Einschätzungen zu Häufigkeiten psychiatrischer Notfälle 19

4.4 Diagnostische Verfahren in der Notaufnahme bei psychisch auffälligen Patienten 26

4.5 Psychopharmakologische Medikamente in der Notaufnahme 27

4.6 Versorgung von psychiatrischen Notfallsituationen in der Notaufnahme 29

5. DISKUSSION 33

5.1 Literaturübersicht 33

5.1.1 Notfallmedizin und Notaufnahme

5.1.2 Der psychiatrische Notfall $\quad 34$

5.1.3 Psychiatrisches Management von psychiatrischen Notfällen 40

$\begin{array}{lll}5.2 & \text { Methodische Fragen } & 42\end{array}$

$\begin{array}{lll}5.3 & \text { Diskussion der Ergebnisse } & 45\end{array}$

6. LITERATURVERZEICHNIS

$\begin{array}{lll}\text { 7. } & \text { ANHANG } & 63\end{array}$ 
7.1 Fragebogen

$\begin{array}{lll}\text { 8. } & \text { PUBLIKATIONEN/DANK } & 78\end{array}$

$\begin{array}{lll}8.1 & \text { Publikationen } & 78\end{array}$

$\begin{array}{lll}8.2 & \text { Danksagungen } & 78\end{array}$

$\begin{array}{lll}9 . & \text { LEBENSLAUF } & 79\end{array}$ 


\section{Zusammenfassung}

\subsection{Zusammenfassung}

Psychiatrische Notfälle und psychisch Auffällige stellen in Notaufnahmen nach klinischer Wahrnehmung einen durchaus relevanten Anteil dar. Wissenschaftliche Untersuchungen zur Häufigkeit dieser Patienten sind nur spärlich vorhanden, Untersuchungen zu Standards in der Diagnostik und Therapie fehlen bisher ganz. Im Sinne einer guten Versorgung psychisch Kranker ist es notwendig zu erfahren, welche Möglichkeiten zur Diagnostik und Behandlung in allgemeinen Notaufnahmen vorhanden sind und wie die ambulante oder stationäre Weiterbehandlung erfolgt.

Wir führten eine Fragebogenuntersuchung an allgemeinen Notaufnahmen im gesamten Bundesgebiet mit Unterstützung der Fachgesellschaften (Deutsche Gesellschaft Interdisziplinäre Notaufnahme e.V. (DGINA), Bundesarbeitsgemeinschaft - Zentrale Notaufnahme (BAG-ZNA)) durch. Darüber hinaus wurden 1073 Krankenhäuser der Grund-, Regel-, Schwerpunkt- und Maximalversorgung angeschrieben und um Teilnahme gebeten. Im Fragebogen sollten Angaben zur Größe der Notaufnahme und der Klinik, zur Häufigkeit definierter psychischer Störungen und Krankheiten sowie zur Möglichkeit diagnostischer und therapeutischer Maßnahmen gemacht werden. Zum Schluss sollte die typische Versorgung anhand von sechs Fallbeispielen dargestellt werden.

74 Notaufnahmen aus dem ganzen Bundesgebiet und aus Kliniken aller Versorgungsstufen nahmen teil, davon $76 \%$ interdisziplinäre Notaufnahmen (durchschnittliche Anzahl Patienten pro Jahr: $22.827 \pm 12.303$, durchschnittliche Anzahl von Klinikbetten: $618 \pm 375$; Psychiatrie als Fach einer interdisziplinären Notaufnahme: 10 (13,5\%), psychiatrische Kompetenz am Standort vorhanden: 84\%). Die Teilnehmer berichteten im Durchschnitt über 15\% psychisch auffällige und $9 \%$ psychiatrisch diagnose- und therapiebedürftige Patienten. Etwa die Hälfte aller psychiatrischen Erkrankungen wurde auf Suchterkrankungen zurückgeführt. Etwa $2 \%$ der Patienten stellten sich nach einem Suizidversuch vor, etwa 3\% der Patienten wurden als aggressiv eingeschätzt. Darüber hinaus wurde über 2,5 $\pm 4,2(0-25)$ verletzte Mitarbeiter durch aggressive Handlungen von Patienten berichtet. $81 \%$ der Notaufnahmen führten gerichtliche Unterbringungen durch, im Mittel $50 \pm 136$ (01.000) pro Jahr. Standardisierte Screeningfragebögen oder 
Selbsteinschätzungsskalen zum Feststellen einer psychischen Erkrankung in der Notaufnahme wurden nur sehr selten, nämlich von 4 Notaufnahmen $(5,4 \%)$ angewendet. Als diagnostische Standards wurden körperliche Untersuchung, die Bestimmung von Blutdruck und Puls sowie die Bestimmung von Glucose, Blutbild, Elektrolyte und Retentionswerten berichtet. Als unverzichtbare Psychopharmaka wurden vor allem Diazepam (91\%), Lorazepam (88\%) und Haloperidol (87\%) genannt.

Ärzte in Notaufnahmen beurteilten etwa 15\% ihrer Patienten als psychisch auffällig, davon ca. $2 / 3$ als psychiatrisch diagnose- und therapiebedürftig. Bezogen auf durchschnittlich 23.000 Patienten pro Jahr bedeutet dies ca. 2.100 psychiatrische Notfallpatienten. Nur in $14 \%$ der Notaufnahmen ist Psychiatrie als eigenständiges Fach vertreten, die meisten Notaufnahmen verfügen aber über eine psychiatrische Kompetenz, z.B. als Konsildienst, am Standort. Als diagnostische Standards werden nur körperliche Untersuchung, die Bestimmung von Blutdruck und Puls und wenige Laboruntersuchungen durchgeführt.

\subsection{Summary}

\section{Standards of care for psychiatric patients in German emergency departments}

Clinicians have the perception that psychiatric emergency situations (PES) and mentally disordered patients are frequently seen in general or medical emergency rooms or departments (EDs). There are, however, only few investigations that focus on the prevalence of these patients in Germany, and none concerning diagnostic and therapeutic standards. PES in EDs should be treated according to comparable standards as medically disabled patients. Thus it is necessary to learn more about the diagnostic and therapeutical possibilities in EDs and about the procedures and the decision-making process whether these patients are transferred to further out- or inpatient treatment.

We conducted a survey in general EDs throughout Germany. Support was received from the two medical associations in which German EDs are organized, the German Society of Inter-Disciplinary Emergency Departments (DGINA), and the National Working Group Hospital Emergency Departments (BAG-ZNA). 1073 level 1-3 hospitals were contacted and asked to participate. The questionnaire consisted of 
questions concerning the size of the ED and of the hospital (e.g. number of patients and physicians), the prevalence of psychiatric disorders, the diagnostic and therapeutic possibilities and standard procedures of dealing with PES, and the way of care in six typical case reports.

Seventy-four EDs that were distributed all over Germany and of all service levels participated (76\% interdisciplinary EDs), with an average of $22,827 \pm 12,303$ patients per year in the ED and $618 \pm 375$ hospital beds. Psychiatry as medical discipline was integrated in 10 EDs (13.5\%), psychiatric competence could be activated in $84 \%$ of EDs. Participating EDs reported prevalence rates of $15 \%$ mentally disordered patients and $9 \%$ of patients that require psychiatric diagnostic and therapeutic procedures. Two percent of all patients presented after suicide attempt, and $3 \%$ were considered to be aggressive. Approximately $50 \%$ of all PES were related to substance abuse disorders. An average of $2.5 \pm 4.2$ (0-25) members of the medical and nursing staff was injured during a one-year period by violent patients. Legal actions against the will of patients were initiated in $81 \%$ of EDs, against an average of $50 \pm 136$ patients per year and ED. Standardized diagnostic screening instruments or self-rating questionnaires were used in only four EDs (5.4\%). As standards (consent of $\geq 80 \%$ of participating centers) for the diagnostic work-up of psychiatric patients (medical clearance) were named physical examination, the measurement of heart rate and blood pressure, and the conduct of some laboratory testing (glucose, blood cell count, electrolytes and renal function). Diazepam (91\%), lorazepam (88\%) und haloperidol $(87 \%)$ were considered as indespensable psychopharmacological agents in the ED.

Physicians in German EDs characterize approximately $15 \%$ of all patients as mentally disordered, two thirds of whom as requiring diagnostic and therapeutical actions. This indicates that approximately 2.100 PES per year require medical help in an average ED with approximately 23,000 patients per year. Psychiatry is integrated to an interdisciplinary ED in only $14 \%$, however, psychiatric competence is available on request in the majority of all EDs. As diagnostic standards in psychiatric patients usually physical examination, the measurement of heart rate and blood pressure, and some laboratory testing are performed. 


\section{Einleitung}

Die Notaufnahme - auch Notfallaufnahme, Notfallambulanz oder Notfallstation genannt - eines Krankenhauses ist die primäre Anlaufstelle zur Akutversorgung von Patienten und stellt einen wichtigen Bestandteil der Notfallmedizin dar. In einer Notaufnahme werden in kurzer Zeit wichtige diagnostische und therapeutische Entscheidungen gefällt, die den Behandlungsverlauf eines akut Erkrankten maßgeblich beeinflussen und deren richtige Einschätzung und Durchführung möglicherweise lebensrettend sein können. Patienten mit einer psychiatrischen Erkrankung oder einer psychiatrischen Koerkrankung stellen in Notaufnahmen für Ärztinnen und Ärzte eine besondere diagnostische und therapeutische Herausforderung dar, da drei wesentliche Faktoren im Umgang mit diesen Patienten zu berücksichtigen sind:

- die Erkennung des Patienten als psychiatrischer Patient

- die schnelle Entscheidung bezüglich akuter therapeutischer Maßnahmen

- die Einschätzung und Beurteilung des Grades an Gefährdung, der aus der psychiatrischen Erkrankung für Patient, Personal und andere Personen ausgehen kann

Untersuchungen zur Häufigkeit und Versorgung psychiatrischer Störungen in deutschen Notaufnahmen sind kaum vorhanden. Die wenigen Untersuchungen deuten aber darauf hin, dass Patienten mit psychiatrischen Erkrankungen in der Notaufnahme deutscher Krankenhäuser keine Seltenheit sind. In einer ersten Untersuchung in Deutschland durch Kropp et al. wurde eine Häufigkeit von 7,7\% festgestellt. Die häufigsten psychiatrischen Diagnosen waren demnach Alkoholintoxikation (20,2\%), paranoide Schizophrenie (14,2\%) und akute Belastungsreaktionen (6,7\%) [28]. Tonn et al. stellten in einer retrospektiven Untersuchung an einem Krankenhaus der Regelversorgung fest, dass nahezu $32 \%$ aller Patienten, die durch Einsatz eines Notarztes der zentralen Notaufnahme zugeführt wurden, unter einer primären psychiatrischen Erkrankung oder einer psychiatrischen Koerkrankung litten [67]. Die Häufigkeit psychiatrischer Notfälle im Notarztdienst in Deutschland beträgt $10-15 \%$ [44]. Kropp et al. wiesen jedoch in ihrer Untersuchung darauf hin, dass Patienten mit psychiatrischen Erkrankungen, die vom Notarzt gesehen werden, nicht identisch mit denen sind, die sich primär in der Notaufnahme eines Krankenhauses vorstellen. In ihrer Untersuchung wurden nur 
$7,8 \%$ aller psychiatrischen Patienten auf Veranlassung von Notärzten in die Notaufnahme eingewiesen [28].

International wurden auch nur wenige Arbeiten publiziert, die sich mit der Diagnosestellung psychiatrischer Erkrankungen in Notaufnahmen beschäftigen. Diese zeigen jedoch fast durchgehend, dass psychiatrische Erkrankungen bei einem erheblichen Anteil dieser Patienten übersehen werden [54, 79]. Zu ausgewählten Krankheitsentitäten ist die Studienlage etwas besser, aber auch hier zeigen die Ergebnisse, dass nur ein geringer Anteil der psychiatrisch Erkrankten primär in der Notaufnahme als solche erkannt wird [30, 48, 54, 59, 73, 79].

Viele Patienten mit einer psychiatrischen Erkrankung sind in einer interdisziplinären Notaufnahme eines Krankenhauses von vornherein als psychiatrisch Erkrankte erkennbar, wie zum Beispiel Patienten mit Suchterkrankungen, mit akuten Entzugsoder Intoxikationssymptomen oder Patienten mit einer akuten Psychose, die durch selbstaggressive oder aggressive Handlungen gegenüber anderen Menschen auffällig geworden sind. Daneben gibt es jedoch viele Patienten, deren psychiatrische Erkrankung oder Komorbidität für psychiatrisch ungeschultes Personal auf den ersten Blick nicht erkennbar ist [54, 79]. Der niedrige Anteil an Patienten, die richtigerweise als psychiatrisch Erkrankte diagnostiziert wurden, kann damit begründet werden, dass nur etwa 10\% dieser Patienten mit der Angabe von Symptomen aus dem psychiatrischen Formenkreis überhaupt vorstellig werden [59, 61]. In interdisziplinären Notaufnahmen gibt es eine Tendenz zur fehlenden Wahrnehmung und Diagnostik psychiatrischer Erkrankungen [2, 14, 67].

Eine systematische Erfassung der Häufigkeit psychischer Störungen in Notaufnahmen deutscher Krankenhäuser und deren Diagnostik- und Behandlungsmöglichkeiten und -gewohnheiten ist bislang noch nicht erfolgt. Ziel dieser wissenschaftlichen Arbeit ist es, den Versorgungsbedarf besser einzuschätzen, um daraus gegebenenfalls Standards für die Diagnostik und Therapie psychiatrischer Erkrankungen in der Notaufnahme entwickeln zu können. 


\section{Material und Methoden}

\subsection{Untersuchungsdesign}

Es wurde eine freiwillige, anonyme Fragebogenuntersuchung an deutschen Krankenhäusern durchgeführt. Der Fragebogen wurde speziell für diese Untersuchung von einem Expertengremium, bestehend aus drei Ärzten aus Notaufnahmen, zwei Psychiatern und zwei Psychologen, konzipiert.

\subsection{Untersuchungsart und Zeitraum}

Der fertiggestellte Fragebogen wurde auf der Website der Deutschen Gesellschaft für Psychiatrie, Psychotherapie und Nervenheilkunde (DGPPN) hinterlegt. Ein Aufruf zur Teilnahme wurde sowohl auf der Website der Deutschen Gesellschaft Interdisziplinäre Notaufnahme e.V. (http://www.dgina.de), als auch auf der Website der Bundesarbeitsgemeinschaft - Zentrale Notaufnahme (http://www.bag-zna.de) hinterlegt, jeweils mit dem nachfolgenden Link: http://www.dgppnberlin.de/index.php?option=com_performs\&ltemid=48. Darüber hinaus wurden im Zeitraum von Dezember 2008 bis Januar 2009 insgesamt 1073 deutsche Krankenhäuser der verschiedenen Versorgungsstufen (Grundversorgung, Regelversorgung, Schwerpunktversorgung, Maximalversorgung), von vorab 1406 ausgesuchten Krankenhäusern, mit der Bitte den Fragebogen auszufüllen, angeschrieben, dabei war vorab nicht klar, welche Krankenhäuser über eine Notaufnahme verfügen. Die Auswahl der Krankenhäuser erfolgte, nach Ausschluss von Rehabilitationskliniken und rein psychiatrischen Kliniken, rein zufällig und willkürlich und ohne Präferenz einer bestimmten geographischen Region. Bei den 1406 Krankenhäusern handelt es sich um sämtliche Krankenhäuser des Bundesgebietes, die uns von den einzelnen Landesärztekammern genannt worden. Nach Ausschluss von Rehabilitationskliniken und rein psychiatrischen Kliniken wurden alle Krankenhäuser mit vollständig vorliegender Adresse angeschrieben. 


\subsection{Fragebogen und Datenerfassung}

Der fünfzehn Seiten umfassende Fragebogen war in ein einleitendes Anschreiben an die ausgesuchten Krankenhäuser und in vier verschiedene Befragungskategorien gegliedert, siehe Anhang.

In dem einleitenden Anschreiben wurde zunächst die Häufigkeit psychischer Störungen in einer interdisziplinären Notaufnahme eines Großklinikums erwähnt sowie auf die Tatsache eingegangen, dass viele psychische Störungen in Notaufnahmen nicht erkannt werden. Des Weiteren wurde darauf hingewiesen, dass eine systematische Erfassung der Häufigkeit psychischer Störungen in Notaufnahmen und deren Diagnostik- und Behandlungsmöglichkeiten bislang noch nicht erfolgte. Zudem wurde kurz die Zielsetzung dieser wissenschaftlichen Untersuchung dargestellt. Auch wurde die Wahrung der Anonymität im Umgang mit den erhobenen Daten zugesichert.

Im ersten Teil des Fragebogens wurde nach den Basisdaten des ausfüllenden Krankenhausmitarbeiters gefragt, nach Alter, Geschlecht und Berufsstatus. Ferner wurden Fragen zum Krankenhaus, wie Postleitzahl des Ortes, in dem sich das Krankenhaus befindet, nach der Versorgungsstufe des Krankenhauses, nach der Bettenzahl der Einrichtung, nach dem Vorhandensein einer psychiatrischen, psychosomatischen oder neurologischen Klinik bzw. eines psychiatrischen Konsildienstes und seiner zeitlichen Verfügbarkeit, gestellt. Daneben wurden speziell Fragen zur Notaufnahme des Krankenhauses gestellt, nach der fachlichen Ausrichtung der Notaufnahme, nach der Anzahl der behandelten Patienten im Zeitraum eines Jahres, nach der Fachrichtung der regulär in der Notaufnahme eingeteilten Ärzte, der Anzahl der diensthabenden Ärzte innerhalb und außerhalb der Regelarbeitszeit und wie viele Ärzte, die fest in der Notaufnahme arbeiten, Facharzt für Psychiatrie (und Psychotherapie) oder Nervenheilkunde sind oder für mindestens ein Jahr in der Psychiatrie und Psychotherapie gearbeitet haben.

Im zweiten Teil des Fragebogens wurde nach geschätzten Häufigkeiten von psychischen Auffälligkeiten und psychiatrischen Notfällen in der Notaufnahme gefragt. Die Angaben sollten in Prozent bezogen auf alle Notfälle eines Referenzzeitraumes von einem Jahr (nach Möglichkeit des Jahres 2007) gemacht werden. Es wurde gefragt: 
- Wie viel Prozent aller Patientinnen und Patienten psychisch auffällig waren.

- Wie viel Prozent der Patientinnen und Patienten für psychiatrisch diagnoseund/oder therapiebedürftig gehalten worden sind.

- Wie viel Prozent der Patientinnen und Patienten an einer psychiatrischen Erkrankung (mit und ohne Suchterkrankungen) litten.

- Wie viel Prozent der Patientinnen und Patienten ein psychiatrisches Konsil erhielten.

- Wie viel Prozent der Patientinnen und Patienten im weitesten Sinne eine psychopharmakologische Medikation erhielten.

- Wie viel Prozent der Patientinnen und Patienten wegen eines Suizidversuchs versorgt worden.

- Wie viel Prozent der Patientinnen und Patienten in der Notaufnahme handgreiflich oder aggressiv geworden sind.

- Wie viele Mitarbeiter/-innen durch aggressive Handlungen von Patientinnen oder Patienten verletzt worden sind.

Ferner wurde gefragt, ob in der Notaufnahme gerichtliche Unterbringungen nach dem Unterbringungsgesetz eingeleitet werden und wie diesbezüglich die Rechtssicherheit eingeschätzt wird.

Im dritten Teil des Fragebogens wurden speziell Fragen zur Diagnostik und Therapie psychischer Erkrankungen gestellt. Es wurde gefragt, ob in der Notaufnahme standardisierte Screeningfragebögen oder Selbsteinschätzungsskalen zum Feststellen einer psychischen Erkrankung bzw. deren Schwere angewendet werden. Es gab folgende Auswahlmöglichkeiten:

- nein, keine

- ja, nämlich

- PRIME-MD

- Patient Health-Questionnaire (PHQ), deutsche Version

- Minimental State Examination (MMSE)

- sonstige

Es wurde gefragt, welche Untersuchungsverfahren bei psychisch auffälligen Patientinnen oder Patienten standardmäßig durchgeführt werden. Folgende Untersuchungsverfahren standen zur Auswahl: 
- körperliche Untersuchung

- Blutdruck- und Pulsbestimmung

- EKG

- Röntgen-Thorax

- Labor

- Blutzuckerbestimmung

- Blutbild

- Leberwerte (z.B. Glutamat-Oxalacetat-Transaminase (GOT), GlutamatPyruvat-Transaminase (GPT), Gamma-Glutamyl-Transferase ( $($-GT))

- Nierenwerte (z.B. Kreatinin, Harnstoff)

- Elektrolyte (z.B. Natrium, Kalium, Kalzium)

- Gerinnung (z.B. Quick, Partielle Thromboplastinzeit)

- Blutgasanalyse

- Pulsoxymetrie

- Drogenscreening

- kranielle Computertomographie (CCT)

- Elektroenzephalographie (EEG)

- Sonstige

Des Weiteren wurde gefragt, welche Medikamente, die zur Behandlung psychiatrischer Störungen in der Notaufnahme geeignet sein könnten, in der Notaufnahme verfügbar sind und welche dieser Medikamente für unverzichtbar gehalten werden, wobei man zwischen oraler und parenteraler Darreichungsform differenzieren konnte. Folgende Medikamente standen zur Auswahl:

- Lorazepam (z.B. Tavor ${ }^{\circledR}$ )

- $\quad$ Diazepam (z.B. Valium ${ }^{\circledR}$ )

- Promethazin (z.B. Atosi ${ }^{\circledR}$ )

- Levomepromazin (z.B. Neurocil ${ }^{\circledR}$ )

- Haloperidol (z.B. Haldol ${ }^{\circledR}$ )

- Amitryptilin (z.B. Saroten ret. ${ }^{\circledR}$, Amineurin $^{\circledR}$ )

- Oxazepam (z.B. Adumbran ${ }^{\circledR}$ )

- Melperon (z.B. Eunerpan ${ }^{\circledR}$ )

- Pipamperon (z.B. Dipiperon ${ }^{\circledR}$ ) 
- weitere

Abschließend konnten die Teilnehmer Medikamente angeben, die in der Notaufnahme darüber hinaus gewünscht werden.

Im vierten Teil des Fragebogens wurde gefragt, wie typischerweise sechs verschiedene psychiatrische Notfallsituationen in der Notaufnahme versorgt werden. Im Einzelnen:

- Schwere, akute Alkoholintoxikation (2,1\%o) eines 28-jährigen ohne größere Störung der Vitalparameter.

- Akuter Verwirrtheitszustand bei einem multimorbiden, hochbetagten Patienten.

- Alkoholentzugsdelir eines typischen 48-jährigen Alkoholabhängigen.

- Suizidversuch einer 32-jährigen Frau durch Einnahme von 10 Tabletten Aspirin und 10 Tabletten Adumbran nach Partnerschaftskonflikt, stark somnolent.

- Stuporöser 62-jähriger Patient ohne erkennbare organische Ursache.

- 28-jährige schizophrene Patientin mit wieder aufgetretenen Halluzinationen und Verfolgungswahn nach unregelmäßiger Einnahme der Medikation.

Folgende Maßnahmen wurden zur Auswahl (Mehrfachwahl möglich) angegeben:

- Medikation

- psychiatrisches Konsil

- stationäre Aufnahme in ein somatisches Krankenhaus

- stationäre Einweisung in ein psychiatrisches Krankenhaus

- körperliche Untersuchung

- Blutdruck- und Pulsbestimmung

- $E K G$

- Röntgen-Thorax

- Labor

- Blutzuckerbestimmung

- Blutbild

- Leberwerte (z.B. GOT, GPT, y-GT)

- Nierenwerte (z.B. Kreatinin, Harnstoff)

- Elektrolyte (z.B. Na, K, Ca)

- Gerinnung (z.B. Quick, PTT) 
- Blutgasanalyse

- Pulsoxymetrie

- Drogenscreening

- kranielle Computertomographie (CCT)

- Elektroenzephalographie (EEG)

- Sonstige

\subsection{Kodierung und Datenerhebung}

Zur Auswertung des Fragebogens wurden die vorgegebenen Antwortmöglichkeiten mit einer aufsteigenden Zahlenreihe kodiert. Bei den Basisdaten wurde zum Beispiel in der Frage nach dem Geschlecht die Antwort "männlich" mit der Ziffer "1" versehen, „weiblich“ mit der Ziffer „2". Bei Fragen, in denen mehrere Vorschläge angegeben wurden, erhielten gleiche Vorschläge gleiche Ziffern. Einzugebende Zahlenwerte wurden direkt übernommen, zum Beispiel Bettenzahl eines Krankenhauses.

\subsection{Statistische Auswertung}

Zur deskriptiven und analytischen Statistik wurden Häufigkeiten und Kreuztabellen mit einem Computersytem (Superior Performing Software Systems (SPSS)) berechnet. Zur statistischen Analyse kamen parametrische und nicht parametrische Testverfahren zur Anwendung.

Unverbundene Gruppen, dass heißt Gruppen ohne Wertigkeit (z.B. Geschlecht) mit 2 Variablen wurden mittels Mann - Whitney - $U$ - Test und Wilcoxon - W - Test auf Unterschiede untersucht und diese mittels des $\mathrm{T}$ - Testes differenziert.

Unverbundene Gruppen mit drei oder mehr Variablen wurden über den KruskalWallis-Test und Chi-Quadrat-Test auf Unterschiede geprüft und diese durch die Oneway-Anover-Auswertung differenziert.

Bei verbundenen Gruppen, d. h. Gruppen mit Wertigkeit sind die Korrelationsanalysen mittels der Funktion nach Spearman (Rangkorrelation) durchgeführt worden.

Das Signifikanzniveau wurde bei mindestens $5 \%(p<0,05)$ festgelegt. 


\section{Ergebnisse}

\subsection{Demographische Daten und Compliance}

Insgesamt wurden 74 ausgefüllte Fragebögen zurückgeschickt. Von den 74 einsendenden Teilnehmern waren 53 männlich $(71,6 \%)$ und 21 weiblich $(28,4 \%)$. Das Alter der einsendenden Ärztinnen und Ärzte betrug durchschnittlich 43,7 $\pm 7,7$ (Minimum: 29, Maximum: 59) Jahre. Von den Teilnehmern waren 32 Fachärzte für Innere Medizin (43,2\%), 15 Fachärzte für Chirurgie (20,3\%), 8 Fachärzte für Anästhesie und Intensivmedizin (10,8\%), 4 Allgemeinmediziner (5,4\%) und 11 waren Fachärzte sonstiger Disziplinen (14,9\%). 4 Teilnehmer machten keine Angaben zu ihrem Facharztstatus (5,4\%).

Aus den angegebenen Postleitzahlen konnten Rückschlüsse auf die Herkunft der Einsender geschlossen werden. Es ergab sich eine breite Verteilung über das gesamte Bundesgebiet.

\subsection{Art der Krankenhäuser und der Notaufnahmen}

Die überwiegende Zahl der Einsendungen kam von Krankenhäusern der Maximalund Schwerpunktversorgung, die über eine Zentrale Notaufnahme (ZNA) verfügen und über 500 Betten bereithalten. Nähere Einzelheiten zur Struktur der Krankenhäuser und ihrer Notaufnahmen finden sich in Tabelle 4.1.

72 Einsender machten Angaben zu der Frage, wie viele Ärztinnen und Ärzte in der Notaufnahme üblicherweise im Dienst sind. In der Regelarbeitszeit sind im Mittel 4,0 $\pm 2,1$ (0-10), außerhalb der Regelarbeitszeit 2,9 $\pm 2,2(0-17)$ Ärztinnen und Ärzte im Dienst.

Auf die Frage, wie viele Ärztinnen und Ärzte, die fest in der Notaufnahme arbeiteten (keine Konsilärzte), Fachärztin/Facharzt für Psychiatrie (und Psychotherapie) oder Nervenheilkunde seien, äußerten sich 71 Einsender. In 23 Notaufnahmen (31,1\%) arbeiteten entweder Fachärzte für Psychiatrie (und Psychotherapie) oder Nervenheilkunde oder Ärztinnen und Ärzte, die mindestens ein Jahr in einer Klinik für Psychiatrie und Psychotherapie gearbeitet hatten (Fachärzte: 12 Notaufnahmen (16,9\%); im Mittel aller Notaufnahmen 0,4 \pm 1,0 (0 - 4); Ärzte mit mindestens 
einjähriger praktischer Erfahrung in der Psychiatrie: 20 Notaufnahmen (30,0\%), im Mittel aller Notaufnahmen 0,6 \pm 1,4 (0-8), 69 Antworten).

\begin{tabular}{|l|l|l|}
\hline Variablen & & Werte \\
\hline Art des Krankenhauses (n, \%) & Maximalversorgung & $24(32,4 \%)$ \\
& Schwerpunktversorgung & $22(29,7 \%)$ \\
& Regelversorgung & $18(24,3 \%)$ \\
& Grundversorgung & $7(9,5 \%)$ \\
& Keine Angabe & $3(4,1 \%)$ \\
\hline Anzahl der Krankenhausbetten & & $618 \pm 375(77-1750)$ \\
(MW \pm SD, Min-Max) & & \\
\hline Art der Notaufnahme (n, \%) & Interdisziplinäre & $56(75,7 \%)$ \\
& Notaufnahme & $10(13,5 \%)$ \\
& Internistische & \\
Anzahl der in der Notaufnahme & Notaufnahme & $7(9,5 \%)$ \\
behandelten Patienten pro Jahr & Chirurgische Notaufnahme & $1(1,4 \%)$ \\
(MW \pm SD, Min-Max) & Keine Angabe & $22.827 \pm 12.303$ \\
\hline Vertretene medizinische Disziplinen & & $(1.500-60.000)$ \\
(n, \%) & Innere Medizin & $62(83,8 \%)$ \\
& & \\
& Unfallchirurgie/Orthopädie & $58(78,4 \%)$ \\
& Allgemeinchirurgie & $49(66,2 \%)$ \\
& Neurologie & $27(36,5 \%)$ \\
& Anästhesie & $22(26,7 \%)$ \\
& Urologie & $15(20,3 \%)$ \\
& Radiologie & $12(16,2 \%)$ \\
& Gynäkologie/Geburtshilfe & $11(14,9 \%)$ \\
& Psychiatrie & $10(13,5 \%)$ \\
& Allgemeinmedizin & $9(12,2 \%)$ \\
& Sonstige & $12(16,2 \%)$ \\
& & \\
\hline
\end{tabular}

Tabelle 4.1: Art der Krankenhäuser und Notaufnahmen 
In Abbildung 4.1 ist dargestellt, in wie weit die teilnehmenden Krankenhäuser über Kliniken oder Abteilungen für Psychiatrie und Psychotherapie, Psychosomatik oder Neurologie verfügen oder ein psychiatrischer Konsildienst verfügbar ist. Bei letzter Frage war der Anteil derjenigen, die dies nicht wussten, bemerkenswert hoch ( $n=5$, $6,8 \%$ ). 58,1\% der Notaufnahmen ( $n=43$ ) gaben an, über keine Klinik für Psychiatrie, Psychotherapie oder Psychosomatik vor Ort zu verfügen. Eine psychiatrische Notaufnahme am Standort wurde von 7 (9,5\%) Krankenhäusern (keine psychiatrische Notaufnahme: $n=36$ (48,6\%); nicht bekannt: $n=31 \quad(41,9 \%))$, eine psychiatrische Aufnahmestation von $13(17,6 \%)$ Einrichtungen berichtet (keine psychiatrische Aufnahmestation: $n=27(36,5 \%)$; nicht bekannt: $n=34(45,9 \%))$.

Bei 83,8\% ( $n=62$ ) war eine psychiatrische Kompetenz, entweder in Form einer psychiatrisch-psychotherapeutischen oder psychosomatischen Klinik oder eines Konsildienstes verfügbar, bei 12 Einrichtungen (16,2\%) bestand diese nicht oder war nicht bekannt.

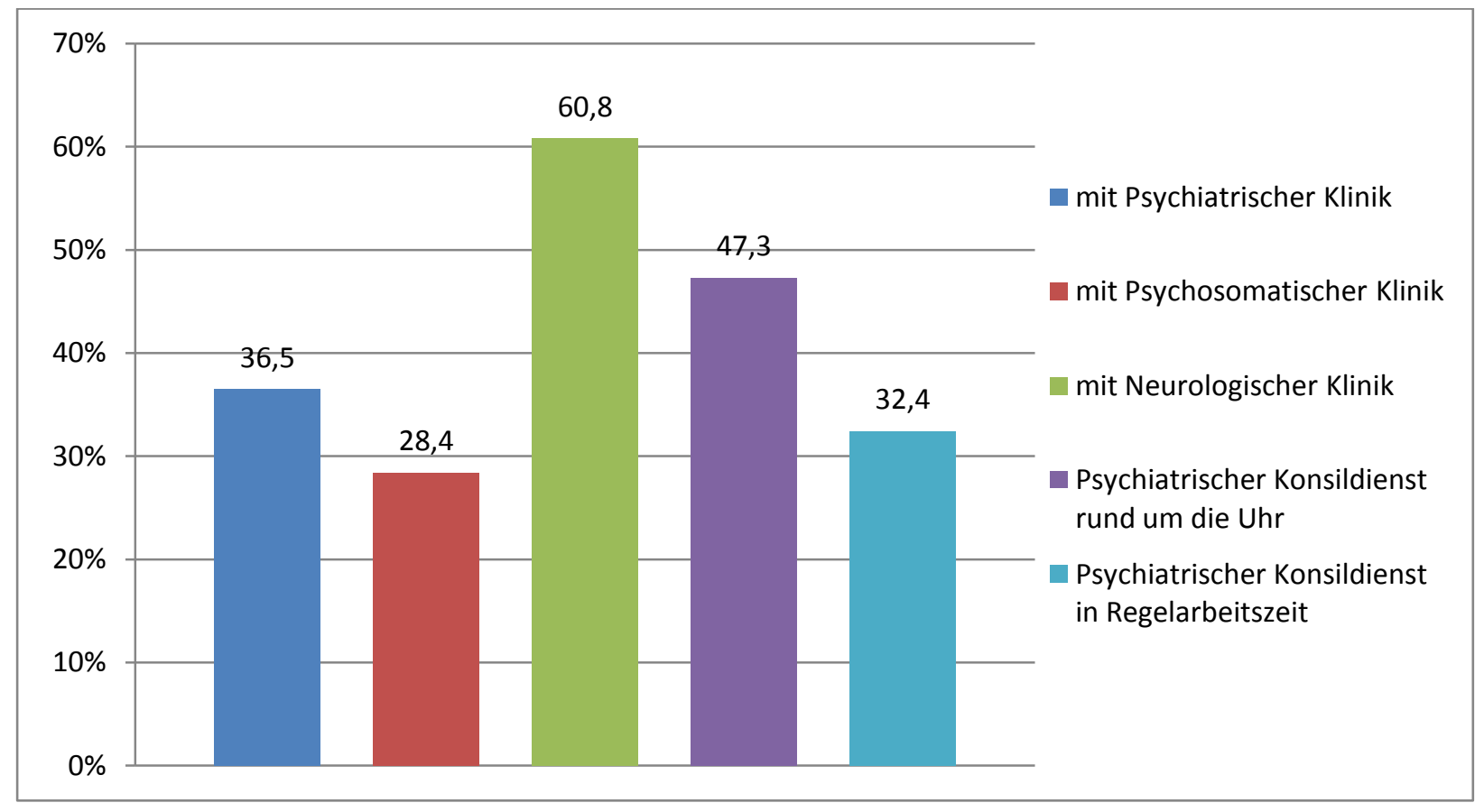

Abbildung 4.1: Krankenhäuser mit Psychiatrischer Kompetenz 


\subsection{Einschätzungen zu Häufigkeiten psychiatrischer Notfälle}

Im Fragebogen fragten wir nach geschätzten Häufigkeiten von psychischen Auffälligkeiten und psychiatrischen Notfällen in der Notaufnahme sowie nach bestimmten diagnostischen und therapeutischen Maßnahmen, bezogen auf alle Notfälle eines Referenzzeitraumes von einem Jahr. Die Ergebnisse finden sich in Tabelle 4.2.

\begin{tabular}{|l|l|}
\hline Variable & Werte ( \%, MW \pm SD, Min-Max) \\
\hline Psychisch auffällig & $15,1 \pm 11,2(0,5-50)$ \\
\hline Psychiatrisch diagnose- und therapiebedürttig & $9,2 \pm 7,6(0-30)$ \\
\hline Psychiatrische Erkrankung (inklusive Suchterkrankungen) & $14,2 \pm 11,8(1,5-65)$ \\
\hline Psychiatrische Erkrankung (exklusive Suchterkrankung) & $6,8 \pm 8,6(0-50)$ \\
\hline Suizidversuche & $1,9 \pm 2,3(0-10)$ \\
\hline Aggressive Patienten & $3,1 \pm 4,0(0-20)$ \\
\hline Psychiatrisches Konsil & $5,2 \pm 7,6(0-50)$ \\
\hline Psychopharmakologische Medikation & $11,1 \pm 11,6(0-70)$ \\
\hline
\end{tabular}

Tabelle 4.2: Häufigkeit psychischer Störungen und bestimmter diagnostischer und therapeutischer Maßnahmen

Die Teilnehmer berichteten über 2,5 \pm 4,2 (0-25) verletzte Mitarbeiter pro Jahr durch aggressive Handlungen von Patienten. Es wurden bis zu 1.000 gerichtliche Unterbringungen nach dem Unterbringungsgesetz (UB) in einer Notaufnahme berichtet, im Mittel 49,8 \pm 135,8 (0 - 1.000). Gerichtliche Unterbringungen wurden in der Mehrzahl nur mit Unterstützung durch einen Psychiater oder Nervenarzt durchgeführt (siehe Abbildung 4.2). Die Rechtssicherheit der Mitarbeiter der Notaufnahmen bei diesen Fragen war nicht sehr hoch (siehe Abbildung 4.3). 


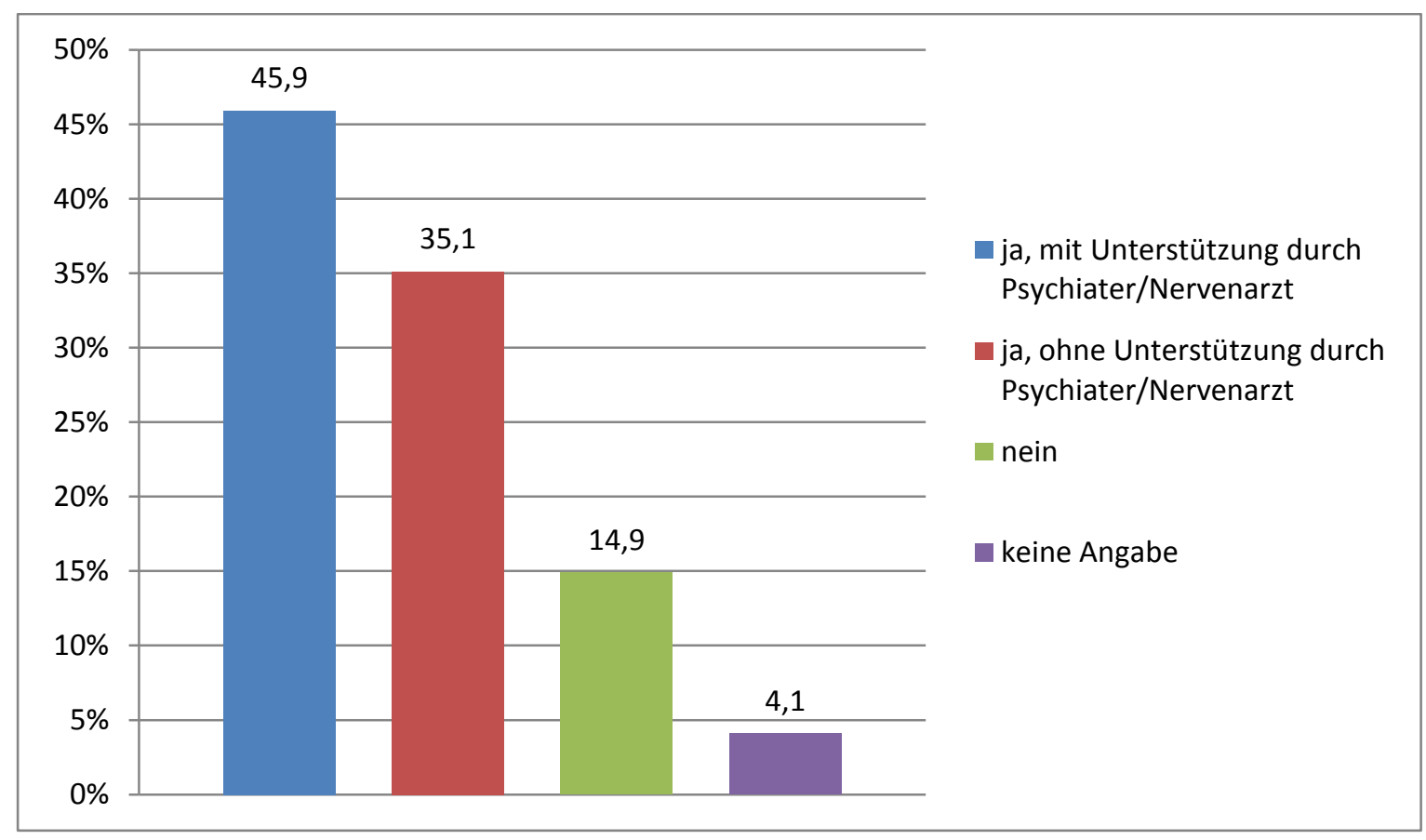

Abbildung 4.2: Gerichtliche Unterbringungen in Notaufnahmen

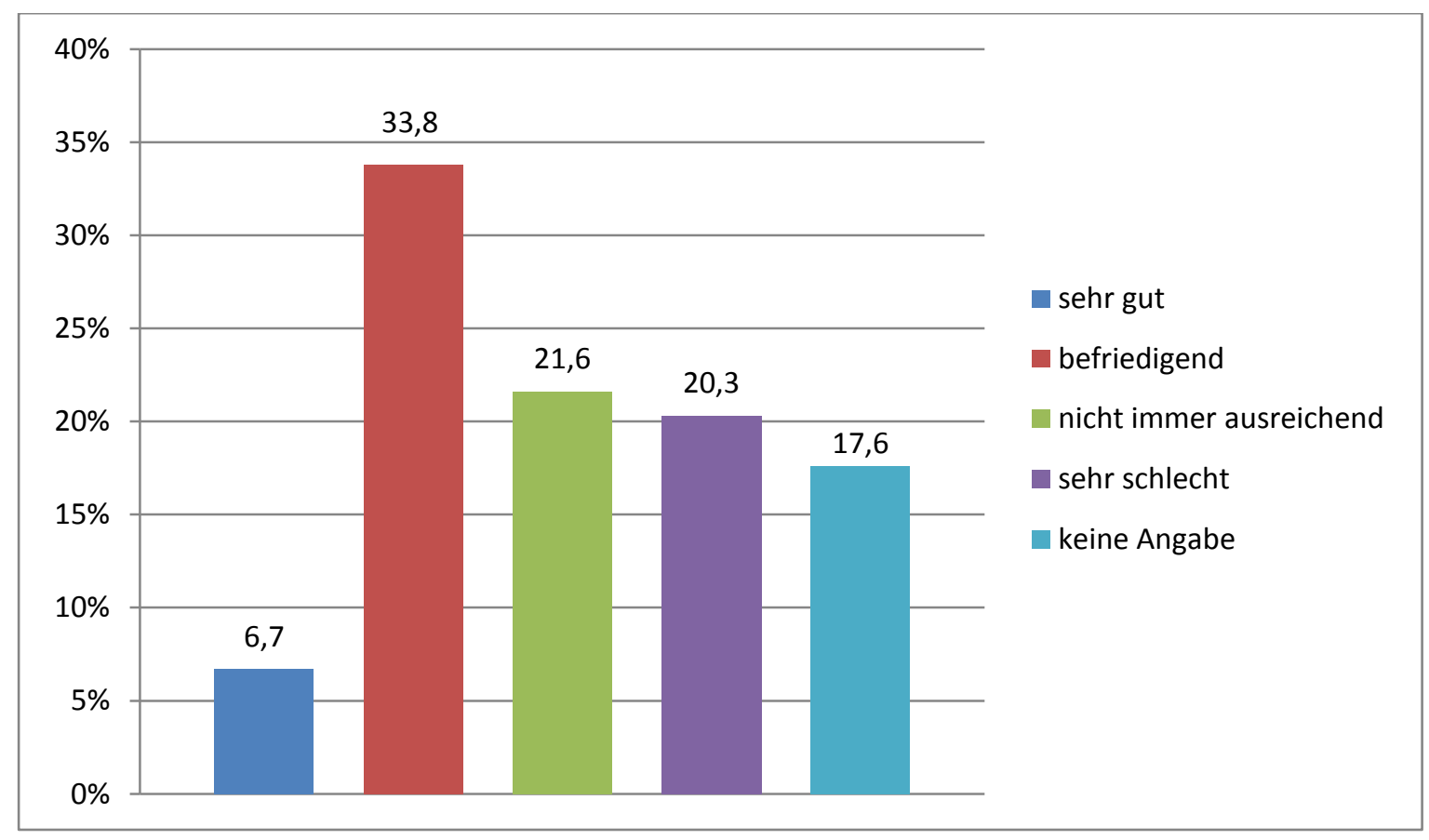

Abbildung 4.3: Rechtssicherheit bei gerichtlichen Unterbringungen 
Die Angaben hinsichtlich der abgefragten psychischen Störungen und Auffälligkeiten bzw. getroffener Maßnahmen korrelieren stark miteinander (siehe Tabelle 4.3). Männer und Frauen beantworteten die Fragen zum Teil unterschiedlich. Frauen gaben bei den meisten Fragen zur Häufigkeit psychischer Störungen in der Notaufnahme höhere Werte an (s. Tabelle 4.4).

In Abhängigkeit von der Art der Notaufnahme fanden sich unterschiedliche Angaben bei den Fragen nach der Häufigkeit psychisch auffälliger Patienten und von Suizidversuchen in der Notaufnahme (Kruskal - Wallis - Test: Häufigkeit psychisch Auffälliger $p=0,047$; interdisziplinäre Notaufnahme: 16,2 $\pm 11,4 \%$, internistische Notaufnahme: $15,4 \pm 12,1 \%$, chirurgische Notaufnahme: 6,3 $\pm 4,8 \%$; Häufigkeit Suizidversuche $p=0,027$, interdisziplinäre Notaufnahme: $1,8 \pm 2,3 \%$, internistische Notaufnahme: $3,2 \pm 2,9, \%$, chirurgische Notaufnahme: $0,7 \pm 0,6 \%$ ).

Beim Vergleich der Versorgungsstufen der Krankenhäuser fand sich lediglich ein Unterschied bei der Häufigkeit psychiatrischer Erkrankungen exklusive Suchterkrankungen, diese wurden in Krankenhäusern der Regelversorgung deutlich häufiger als in Krankenhäusern der Maximalversorgung berichtet (Kruskal - Wallis Test $p=0,012$; Krankenhaus der Maximalversorgung: 3,9 $\pm 4,2 \%$, Krankenhaus der Schwerpunktversorgung: 8,1 \pm 10,6\%, Krankenhaus der Regelversorgung: $10.0 \pm$ 11,4\%, Krankenhaus der Grundversorgung: 4,9 $\pm 3,5 \%$ ).

Notaufnahmen, an deren Standort sich eine psychiatrische oder eine psychosomatische Klinik befand, berichteten über signifikant weniger Suizidversuche, aber über eine höhere Anzahl gerichtlicher Unterbringungen (nur grenzwertig signifikant) und eine höhere Anzahl verletzter Mitarbeiter. Hinsichtlich der berichteten Häufigkeiten für alle anderen abgefragten Variablen ergab sich kein Unterschied in Abhängigkeit von dem Vorhandensein einer psychiatrischen oder psychosomatischen Klinik am Standort. Die Anwesenheit einer neurologischen Klinik am Krankenhaus spielte keine signifikante Rolle bei der Angabe der Häufigkeiten unterschiedlicher psychischer Störungen oder Auffälligkeiten bzw. getroffener Maßnahmen. Bestand die Möglichkeit eines psychiatrischen Konsils, so wurden die Häufigkeit psychiatrischer Erkrankungen einschließlich Suchterkrankungen und die Häufigkeit von Suizidversuchen in der Notaufnahme signifikant geringer angegeben. War eine psychiatrische Aufnahmestation im Krankenhaus vorhanden, so war die Häufigkeit psychischer Erkrankungen einschließlich und exklusive 
Suchterkrankungen und die Häufigkeit aggressiver Patienten in der Notaufnahme geringer. Das Vorhandensein einer psychiatrischen Notaufnahme hatte keinen Einfluss auf die berichteten Häufigkeiten für psychische Störungen und Auffälligkeiten bzw. getroffene Maßnahmen (siehe Tabelle 4.5). 


\begin{tabular}{|c|c|c|c|c|c|c|c|c|}
\hline & $\begin{array}{l}\text { Psychisch } \\
\text { auffällig }\end{array}$ & $\begin{array}{l}\text { Psychiatrisch } \\
\text { diagnose- und } \\
\text { therapiebedürftig }\end{array}$ & $\begin{array}{l}\text { Psychiatrische } \\
\text { Erkrankung } \\
\text { einschließlich } \\
\text { Sucht }\end{array}$ & $\begin{array}{l}\text { Psychiatrische } \\
\text { Erkrankung } \\
\text { exklusive } \\
\text { Sucht }\end{array}$ & $\begin{array}{l}\text { Psychiatrisches } \\
\text { Konsil }\end{array}$ & $\begin{array}{l}\text { Psychopharma- } \\
\text { kologische } \\
\text { Medikation }\end{array}$ & Suizidversuch & $\begin{array}{l}\text { Aggressive } \\
\text { Patienten }\end{array}$ \\
\hline Psychisch auffällig & 1 & & & & & & & \\
\hline $\begin{array}{l}\text { Psychiatrisch diagnose- und } \\
\text { therapiebedürttig }\end{array}$ & $\begin{array}{l}, 741 \\
0,000\end{array}$ & 1 & & & & & & \\
\hline $\begin{array}{l}\text { Psychiatrische Erkrankung einschließlich } \\
\text { Sucht }\end{array}$ & $\begin{array}{l}, 656 \\
0,000\end{array}$ & $\begin{array}{l}, 673 \\
0,000\end{array}$ & 1 & & & & & \\
\hline Psychiatrische Erkrankung exklusive Sucht & $\begin{array}{l}, 556 \\
0,000\end{array}$ & $\begin{array}{l}, 632 \\
0,000\end{array}$ & $\begin{array}{l}, 760 \\
0,000\end{array}$ & 1 & & & & \\
\hline Psychiatrisches Konsil & $\begin{array}{l}, 440 \\
0,000\end{array}$ & $\begin{array}{l}, 609 \\
0,000\end{array}$ & $\begin{array}{l}, 389 \\
0,001\end{array}$ & $\begin{array}{l}, 711 \\
0,000\end{array}$ & 1 & & & \\
\hline Psychopharmakologische Medikation & $\begin{array}{l}, 358 \\
0,003\end{array}$ & $\begin{array}{l}, 459 \\
0,000\end{array}$ & $\begin{array}{l}, 331 \\
0,006\end{array}$ & $\begin{array}{l}, 149 \\
0,228\end{array}$ & $\begin{array}{l}, 138 \\
0,270\end{array}$ & 1 & & \\
\hline Suizidversuch & $\begin{array}{l}, 247 \\
0,041\end{array}$ & $\begin{array}{l}, 339 \\
0,004\end{array}$ & $\begin{array}{l}, 374 \\
0,002\end{array}$ & $\begin{array}{l}, 178 \\
0,144\end{array}$ & $\begin{array}{l}, 118 \\
0,338\end{array}$ & $\begin{array}{l}, 321 \\
0,009\end{array}$ & 1 & \\
\hline Aggressive Patienten & $\begin{array}{l}, 279 \\
0,020\end{array}$ & $\begin{array}{l}, 288 \\
0,016\end{array}$ & $\begin{array}{l}, 439 \\
0,000\end{array}$ & $\begin{array}{l}, 184 \\
0,131\end{array}$ & $\begin{array}{l}, 099 \\
0,422\end{array}$ & $\begin{array}{l}, 148 \\
0,237\end{array}$ & $\begin{array}{l}, 287 \\
0,017\end{array}$ & 1 \\
\hline
\end{tabular}

Tabelle 4.3: Korrelationen zur Angabe der Häufigkeiten psychischer Störungen und einzelner Maßnahmen (Korrelationskoeffizient und Signifikanzniveau) 


\begin{tabular}{|c|c|c|c|}
\hline Variable & Geschlecht & Werte (\%) & T-Test (F, p) \\
\hline \multirow[t]{2}{*}{ psychisch auffällig } & Männer & $13,9 \pm 9,1$ & 10,211 \\
\hline & Frauen & $18,3 \pm 15,1$ & 0,002 \\
\hline \multirow{2}{*}{$\begin{array}{l}\text { Psychiatrisch diagnose - und } \\
\text { therapiebedürftig }\end{array}$} & Männer & $8,4 \pm 5,5$ & 14,900 \\
\hline & Frauen & $11,3 \pm 11,1$ & 0,000 \\
\hline \multirow{2}{*}{$\begin{array}{l}\text { Psychiatrische Erkrankungen inklusive } \\
\text { Suchterkrankungen }\end{array}$} & Männer & $12,9 \pm 7,9$ & 13,846 \\
\hline & Frauen & $17,5 \pm 18,1$ & 0,000 \\
\hline \multirow{2}{*}{$\begin{array}{l}\text { Psychiatrische Erkrankungen exklusive } \\
\text { Suchterkrankungen }\end{array}$} & Männer & $5,7 \pm 3,8$ & 21,256 \\
\hline & Frauen & $9,5 \pm 15,0$ & 0,000 \\
\hline \multirow[t]{2}{*}{ Psychiatrisches Konsil } & Männer & $4,3 \pm 4,6$ & 8,462 \\
\hline & Frauen & $7,5 \pm 12,4$ & 0,005 \\
\hline \multirow[t]{2}{*}{ Psychopharmakologische Medikation } & Männer & $12,0 \pm 12,7$ & 1,279 \\
\hline & Frauen & $8,9 \pm 8,1$ & 0,26 \\
\hline \multirow[t]{2}{*}{ Suizidversuche } & Männer & $1,9 \pm 2,4$ & 0,172 \\
\hline & Frauen & $1,8 \pm 2,0$ & 0,68 \\
\hline \multirow[t]{2}{*}{ Aggressive Patienten } & Männer & $2,7 \pm 3,7$ & 4,430 \\
\hline & Frauen & $4,2 \pm 4,7$ & 0,039 \\
\hline
\end{tabular}

Tabelle 4.4: Unterschiede im Antwortverhalten in Abhängigkeit vom Geschlecht des Antwortenden 


\begin{tabular}{|c|c|c|c|}
\hline Variable & Abhängige Variable & Werte (\%) & T-Test (F, p) \\
\hline \multirow[t]{6}{*}{ Suizidversuche } & Psychiatrische Klinik am Standort & $1,3 \pm 1,3$ & 6,136 \\
\hline & Keine psychiatrische Klinik am Standort & $2,2 \pm 2,7$ & 0,016 \\
\hline & psychosomatische Klinik am Standort & $1,0 \pm 1,1$ & 6,931 \\
\hline & Keine psychosomatische Klinik am Standort & $2,3 \pm 2,6$ & 0,011 \\
\hline & Psychiatrisches Konsil möglich & $1,7 \pm 2,1$ & 8,619 \\
\hline & Kein psychiatrisches Konsil möglich & $3,6 \pm 3,7$ & 0,005 \\
\hline \multirow{4}{*}{$\begin{array}{l}\text { Psychiatrische } \\
\text { Erkrankungen } \\
\text { einschließlich } \\
\text { Suchterkrankungen }\end{array}$} & Psychiatrisches Konsil möglich & $13,7 \pm 10,5$ & 5,136 \\
\hline & Kein psychiatrisches Konsil möglich & $19,4 \pm 18,7$ & 0,027 \\
\hline & $\begin{array}{l}\text { Psychiatrische Aufnahmestation am } \\
\text { Krankenhaus }\end{array}$ & $12,2 \pm 4,3$ & $\begin{array}{l}7,341 \\
0,010\end{array}$ \\
\hline & $\begin{array}{l}\text { Keine psychiatrische Aufnahmestation am } \\
\text { Krankenhaus }\end{array}$ & $16,8 \pm 13,4$ & \\
\hline \multirow{2}{*}{$\begin{array}{l}\text { Psychiatrische } \\
\text { Erkrankungen } \\
\text { exklusive } \\
\text { Suchterkrankungen }\end{array}$} & $\begin{array}{l}\text { Psychiatrische Aufnahmestation am } \\
\text { Krankenhaus }\end{array}$ & $5,3 \pm 2,5$ & \multirow[t]{2}{*}{$\begin{array}{l}4,265 \\
0,046\end{array}$} \\
\hline & $\begin{array}{l}\text { Keine psychiatrische Aufnahmestation am } \\
\text { Krankenhaus }\end{array}$ & $10,1 \pm 12,7$ & \\
\hline \multirow{2}{*}{$\begin{array}{l}\text { Anzahl } \\
\text { gerichtlicher } \\
\text { Unterbringungen }\end{array}$} & $\begin{array}{l}\text { Psychiatrische oder psychosomatische Klinik } \\
\text { am Standort }\end{array}$ & $88,5 \pm 197,8$ & \multirow[t]{2}{*}{$\begin{array}{l}8,301 \\
0,088\end{array}$} \\
\hline & $\begin{array}{l}\text { Keine psychiatrische oder psychosomatische } \\
\text { Klinik am Standort }\end{array}$ & $19,4 \pm 25,9$ & \\
\hline \multirow[t]{2}{*}{$\begin{array}{l}\text { Aggressive } \\
\text { Patienten }\end{array}$} & $\begin{array}{l}\text { Psychiatrische Aufnahmestation am } \\
\text { Krankenhaus }\end{array}$ & $2,3 \pm 1,9$ & \multirow[t]{2}{*}{$\begin{array}{l}5,996 \\
0,019\end{array}$} \\
\hline & $\begin{array}{l}\text { Keine psychiatrische Aufnahmestation am } \\
\text { Krankenhaus }\end{array}$ & $4,2 \pm 5,3$ & \\
\hline \multirow[t]{2}{*}{$\begin{array}{l}\text { Anzahl verletzte } \\
\text { Mitarbeiter }\end{array}$} & $\begin{array}{l}\text { Psychiatrische oder psychosomatische Klinik } \\
\text { am Standort }\end{array}$ & $3,9 \pm 5,7$ & \multirow[t]{2}{*}{$\begin{array}{l}11,250 \\
0,022\end{array}$} \\
\hline & $\begin{array}{l}\text { Keine psychiatrische oder psychosomatische } \\
\text { Klinik am Standort }\end{array}$ & $1,4 \pm 1,8$ & \\
\hline
\end{tabular}

Tabelle 4.5: Unterschiede in Abhängigkeit vom Vorhandensein psychiatrischer Kompetenz 


\subsection{Diagnostische Verfahren in der Notaufnahme bei psychisch auffälligen Patienten}

Standardisierte Screeningfragebögen oder Selbsteinschätzungsskalen zum Feststellen einer psychischen Erkrankung in der Notaufnahme werden nur sehr selten, nämlich von 4 Notaufnahmen (5,4\%) angewendet (keine Angabe: $n=5,6,8 \%$ ).

Folgende Untersuchungsverfahren wurden bei psychisch auffälligen Patientinnen und Patienten standardmäßig in der Notaufnahme angewendet, selbst wenn keine klare organische Ursache erkennbar war (Tabelle 4.6). Von je einer Notaufnahme $(1,4 \%)$ wurden darüber hinaus die routinemäßige Bestimmung des Medikamentenspiegels und die Thyreotropinbestimmung (TSH) genannt.

\begin{tabular}{|l|l|}
\hline Untersuchungsverfahren & Wert (n, \%) \\
\hline körperliche Untersuchung & $72(97,3)$ \\
\hline Blutdruck- und Pulsbestimmung & $72(97,3)$ \\
\hline Elektrokardiogramm (EKG) & $57(77,0)$ \\
\hline Röntgenaufnahme Thorax & $12(16,2)$ \\
\hline Pulsoxymetrie & $46(62,2)$ \\
\hline Drogenscreening & $24(32,4)$ \\
\hline kranielle Computertomographie (CCT) & $19(25,7)$ \\
\hline Elektroenzephalographie (EEG) & $1(1,4)$ \\
\hline Laborbestimmungen: & \\
\hline Glukose & $69(93,2)$ \\
\hline Blutbild & $65(87,8)$ \\
\hline Leberwerte (z.B. GOT, GPT, Y-GT) & $54(73,0)$ \\
\hline Nierenwerte (z.B. Kreatinin, Harnstoff) & $62(83,8)$ \\
\hline Elektrolyte (z.B. Na, K, Ca) & $64(86,5)$ \\
\hline Gerinnung (z.B. Quick, PTT) & $50(67,6)$ \\
\hline Blutgasanalyse & $12(16,2)$ \\
\hline
\end{tabular}

Tabelle 4.6: Standardmäßig durchgeführte Untersuchungsverfahren bei psychiatrischen Patienten 
Hinsichtlich der diagnostischen Standards fand sich kein Unterschied, ob an den Standorten eine psychiatrische oder psychosomatische Klinik vorhanden war oder nicht. In Notaufnahmen an Standorten mit psychiatrischer Kompetenz (Klinik oder Konsildienst) wurde häufiger eine Analyse der Gerinnungsparameter durchgeführt (45/62 vs. $\left.5 / 12, \mathrm{Chi}^{2} \mathrm{df}=1 ; \mathrm{p}=0.048\right)$.

\subsection{Psychopharmakologische Medikamente in der Notaufnahme}

In Abbildung 4.4 sind die von den Teilnehmern genannten verfügbaren Psychopharmaka in oraler und parenteraler Darreichungsform in den Notaufnahmen dargestellt. Unter den Benzodiazepinen sind Lorazepam und Diazepam in den meisten Notaufnahmen oral, Diazepam auch parenteral vorhanden. Haloperidol ist häufiger parenteral als oral vorrätig, Promethazin sehr viel häufiger als Levomepromazin.

Verblüffend waren die Angaben zu Oxazepam und Pipamperon. Hier fragten wir als Kontrolle auch die Verfügbarkeit der parenteralen Form ab, obwohl es diese in Deutschland gar nicht gibt.

Als weitere verfügbare Medikamente, die zur Behandlung psychiatrischer Störungen in der Notaufnahme geeignet sein könnten, wurden genannt: Clonidin ( $n=4 ; 5,5 \%)$, Clomethiazol $(n=3 ; 4,1 \%)$, Midazolam $(n=1 ; 1,4 \%)$, Olanzapin $(n=1 ; 1,4 \%)$, Carbamazepin $(n=1 ; 1,4 \%)$, Dikaliumclorazepat $(n=1 ; 1,4 \%)$ sowie Trimipramin $(n=1$; $1,4 \%)$.

Auf die Frage, welche der genannten Medikamente in der Notaufnahme unverzichtbar seien, wurden vor allem Diazepam, Lorazepam und Haloperidol genannt. Als am ehesten verzichtbar wurden Oxazepam, Amitryptilin und Pipamperon angegeben. In der Tabelle 4.7 werden die Ergebnisse zusammenfassend wiedergegeben. 


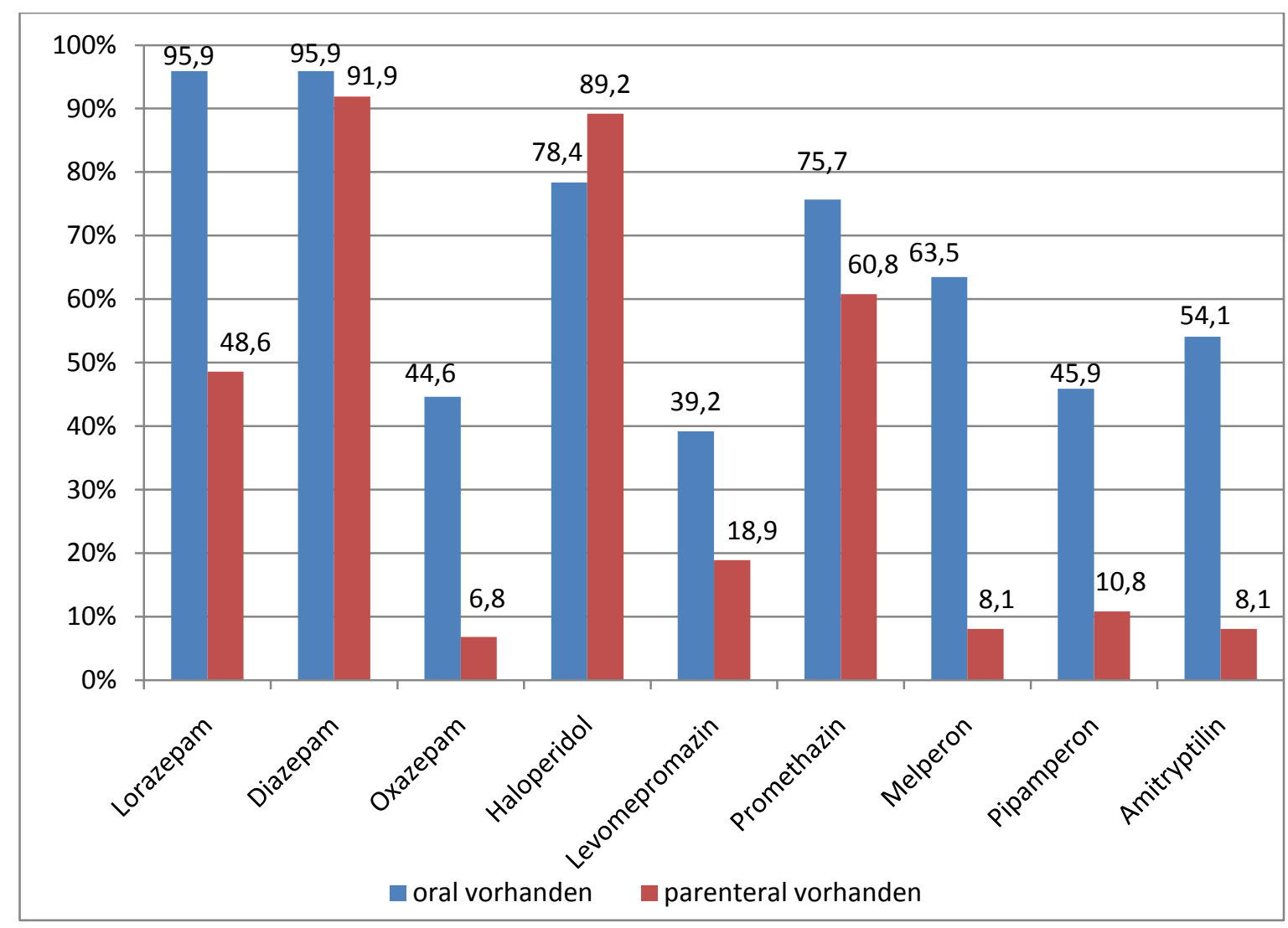

Abbildung 4.4: verfügbare Psychopharmaka in der Notaufnahme

\begin{tabular}{|l|l|l|l|l|}
\hline Präparat & unverzichtbar & $\begin{array}{l}\text { oral } \\
\text { unverzichtbar }\end{array}$ & $\begin{array}{l}\text { parenteral } \\
\text { unverzichtbar }\end{array}$ & $\begin{array}{l}\text { keine } \\
\text { Angabe }\end{array}$ \\
\hline Lorazepam & $65(87,8 \%)$ & $60(81,1 \%)$ & $29(39,2 \%)$ & $9(12,2 \%)$ \\
\hline Diazepam & $67(90,5 \%)$ & $45(60,8 \%)$ & $62(83,8 \%)$ & $7(9,5 \%)$ \\
\hline Promethazin & $36(48,6 \%)$ & $33(44,6 \%)$ & $21(28,4 \%)$ & $38(51,4 \%)$ \\
\hline Levomepromazin & $15(20,3 \%)$ & $11(14,9 \%)$ & $8(10,8 \%)$ & $59(79,7 \%)$ \\
\hline Haloperidol & $64(86,5 \%)$ & $41(55,4 \%)$ & $57(77,0 \%)$ & $10(13,5 \%)$ \\
\hline Amitryptilin & $8(10,8 \%)$ & $8(10,8 \%)$ & $2(2,7 \%)$ & $66(89,2 \%)$ \\
\hline Oxazepam & $5(6,8 \%)$ & $5(6,8 \%)$ & $2(2,7 \%)$ & $69(93,2 \%)$ \\
\hline Melperon & $21(28,4 \%)$ & $20(27,0 \%)$ & $4(5,4 \%)$ & $53(71,6 \%)$ \\
\hline Pipamperon & $14(18,9 \%)$ & $12(16,2 \%)$ & $6(8,1 \%)$ & $60(81,1 \%)$ \\
\hline
\end{tabular}

Tabelle 4.7: Unverzichtbare Psychopharmaka in der Notaufnahme, Angaben absolut (n) und in Prozent (\%) 
In Abhängigkeit davon, ob eine psychiatrische oder psychosomatische Klinik am Standort vorhanden ist, bestehen keine Unterschiede hinsichtlich der Verfügbarkeit aller genannten Psychopharmaka in den Notaufnahmen. Gleiches ergab sich in Abhängigkeit von der Frage nach dem Vorhandensein psychiatrischer Kompetenz am Standort. Bei der Frage nach der Beurteilung der Unverzichtbarkeit von Psychopharmaka ergaben sich lediglich einige grenzwertige Unterschiede. An Standorten, an denen eine psychiatrische oder psychosomatische Klinik vorhanden ist, wird Lorazepam eher für unverzichtbar gehalten (30/31 vs. $35 / 42 ; \mathrm{Chi}^{2} \mathrm{df}=1$; $\mathrm{p}=0.071)$. Dies gilt ähnlich für Standorte mit psychiatrischer Kompetenz (57/62 vs. 8/11; $\left.\mathrm{Chi}^{2} \mathrm{df}=1 ; \mathrm{p}=0.094\right)$; an diesen Standorten wird Amitryptilin tendenziell eher für verzichtbar gehalten ( $\left.57 / 62 \mathrm{vs.} 8 / 11 ; \mathrm{Chi}^{2} \mathrm{df}=1 ; \mathrm{p}=0.094\right)$.

\subsection{Versorgung von psychiatrischen Notfallsituationen in der Notaufnahme}

Im vierten Teil des Fragebogens wurde gefragt, wie typischerweise sechs verschiedene psychiatrische Notfallsituationen in der Notaufnahme versorgt werden. Es wurde ein Maßnahmenkatalog zur Auswahl gestellt, wobei Mehrfachwahl möglich war. Die Ergebnisse werden in den Abbildungen 4.5 bis 4.9 zusammenfassend dargestellt.

In Abhängigkeit davon, ob eine psychiatrische oder psychosomatische Klinik am Standort vorhanden ist, wurden die Fallbeispiele teils unterschiedlich bearbeitet. Demnach würde der hochbetagte Patient mit Verwirrtheitszustand häufiger in eine psychiatrische Klinik eingewiesen werden, wenn diese am Standort vorhanden ist (14/31 vs. 8/43; $\left.\mathrm{Chi}^{2} \mathrm{df}=2 ; \mathrm{p}=0.046\right)$, ebenso der delirante Alkoholabhängige (19/31 vs. 12/43; $\left.\mathrm{Chi}^{2} \mathrm{df}=2 ; \mathrm{p}=0.014\right)$. Der stuporöse Patient würde seltener im somatischen Krankenhaus (15/31 vs. 34/43; $\mathrm{Chi}^{2} \mathrm{df}=2 ; \mathrm{p}=0.021$ ), dafür häufiger in der Psychiatrie stationär (17/31 vs. 8/43; $\left.\mathrm{Chi}^{2} \mathrm{df}=2 ; \mathrm{p}=0.005\right)$ aufgenommen werden. Keine Unterschiede ergaben sich bei dieser Fragestellung in Abhängigkeit davon, ob eine psychiatrische Kompetenz (Klinik oder Konsildienst) vorhanden war oder nicht. 


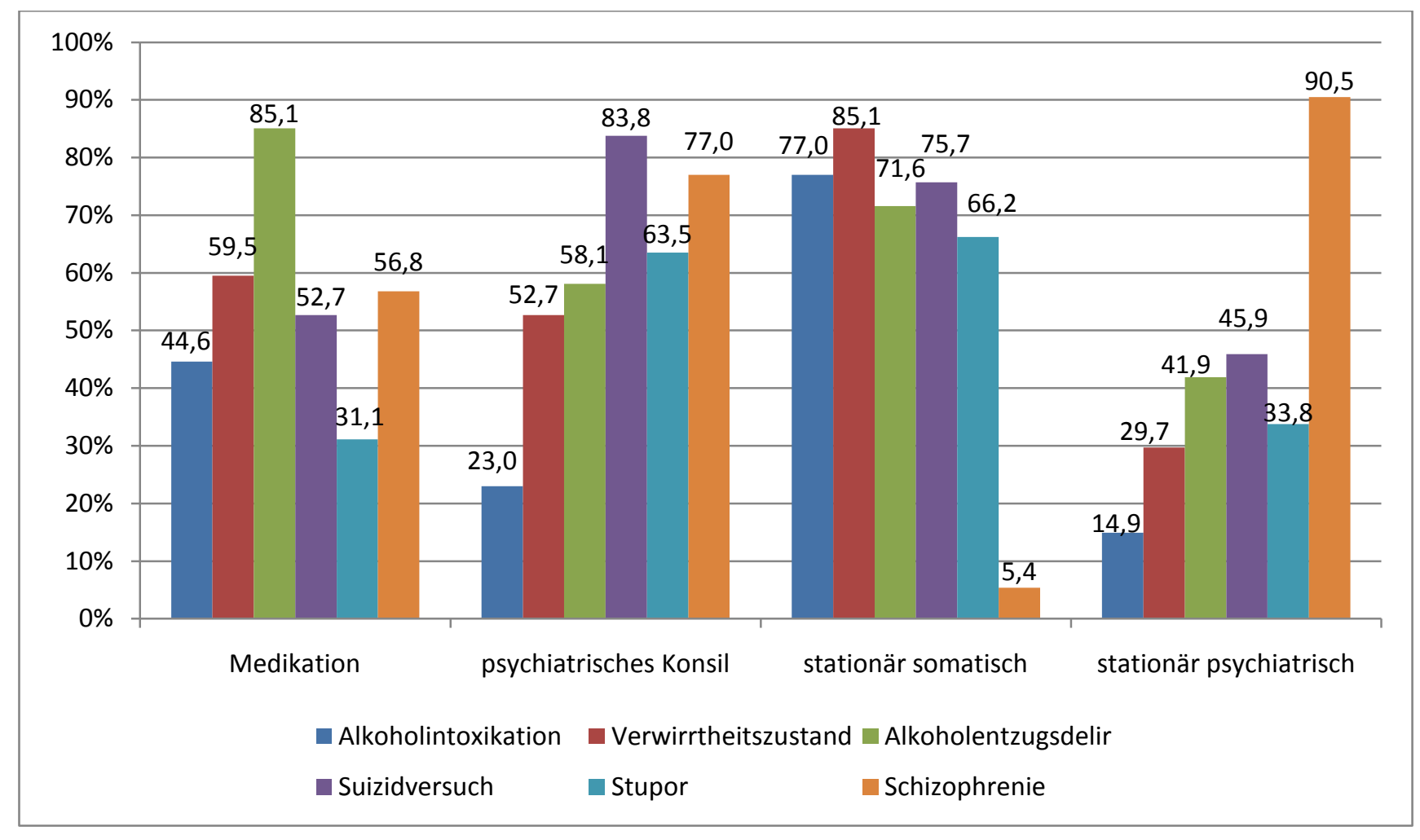

Abbildung 4.5: Versorgung von psychiatrischen Notfallsituationen in der Notaufnahme (1)

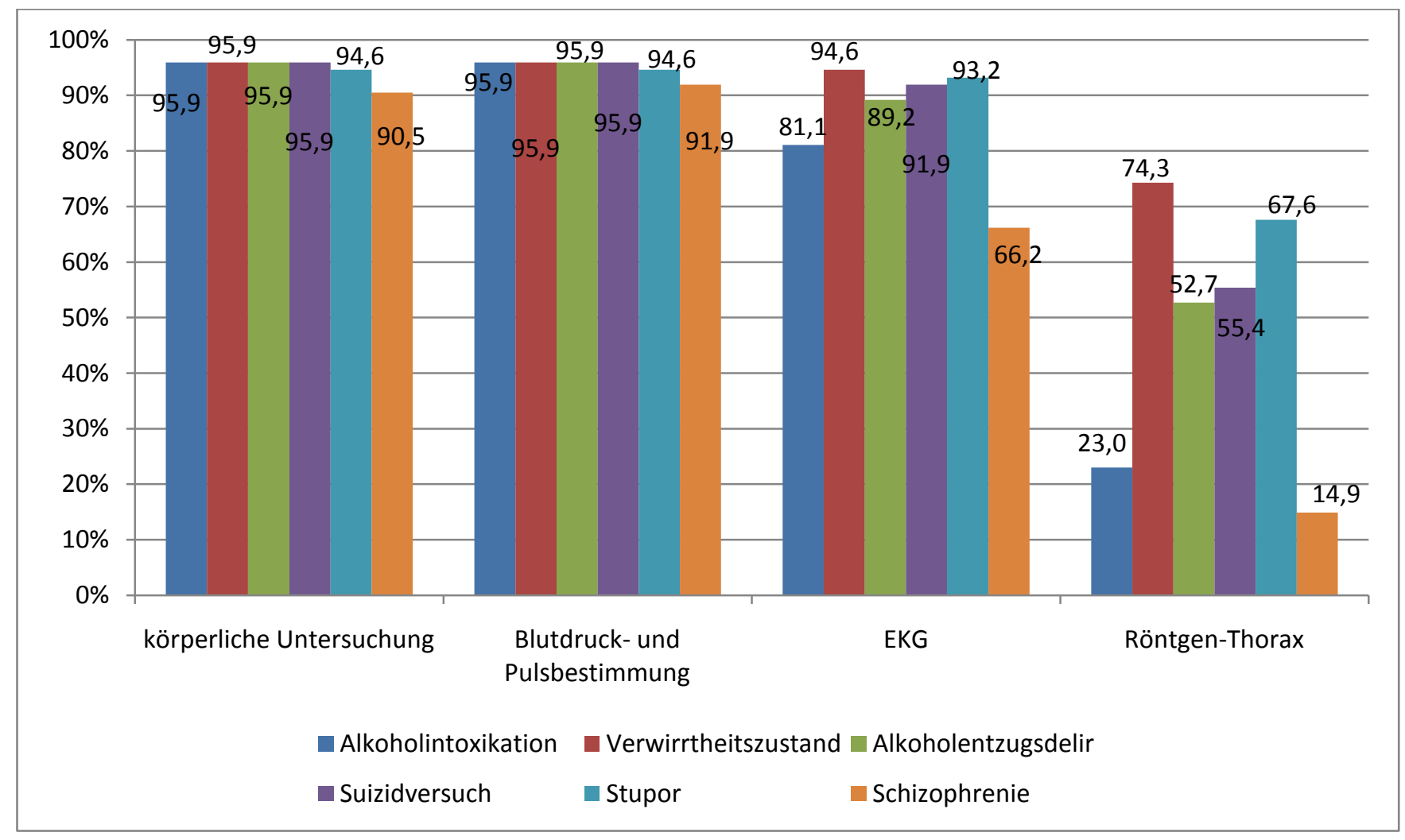

Abbildung 4.6: Versorgung von psychiatrischen Notfallsituationen in der Notaufnahme (2) 


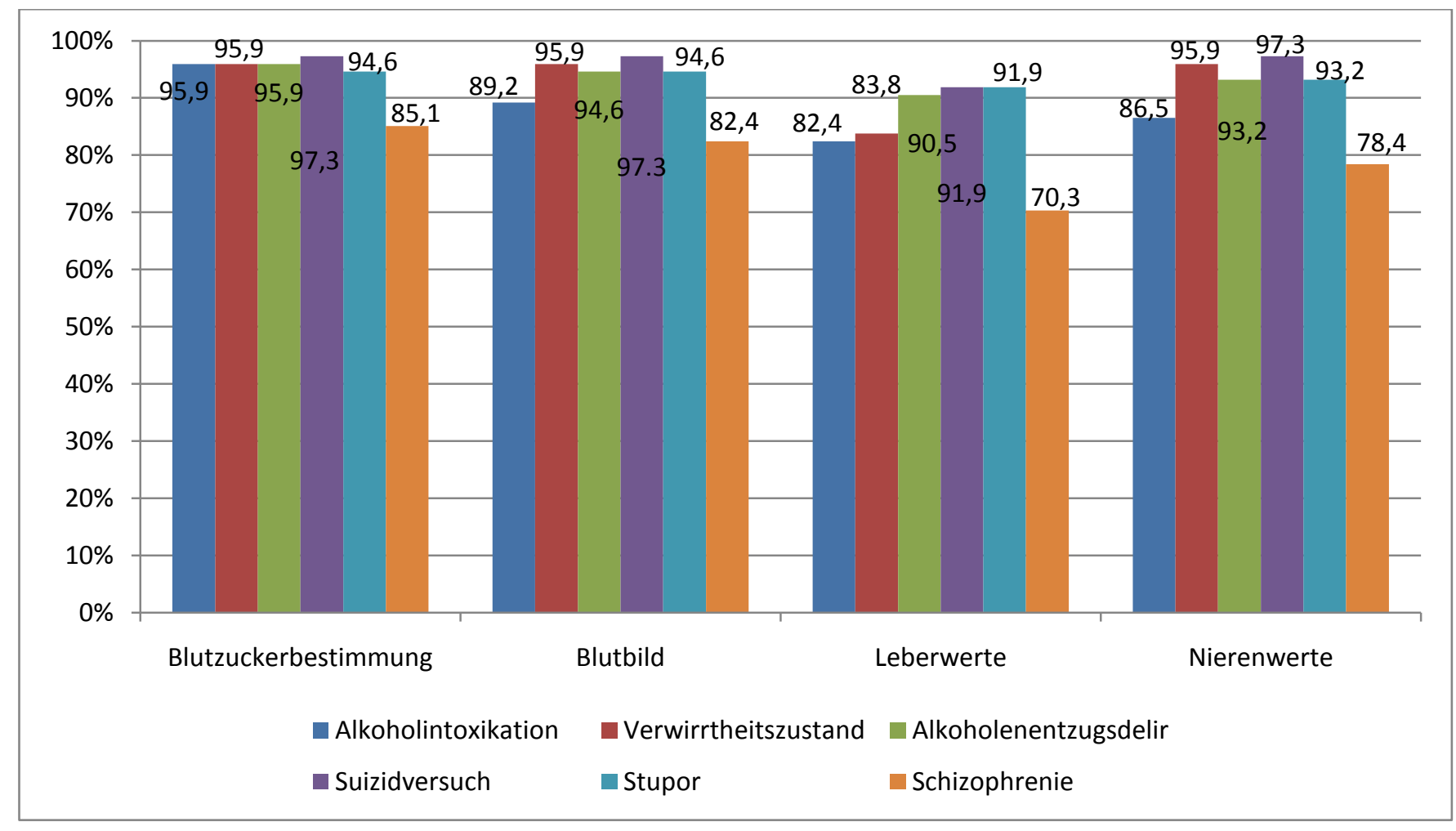

Abbildung 4.7: Versorgung von psychiatrischen Notfallsituationen in der Notaufnahme (3)

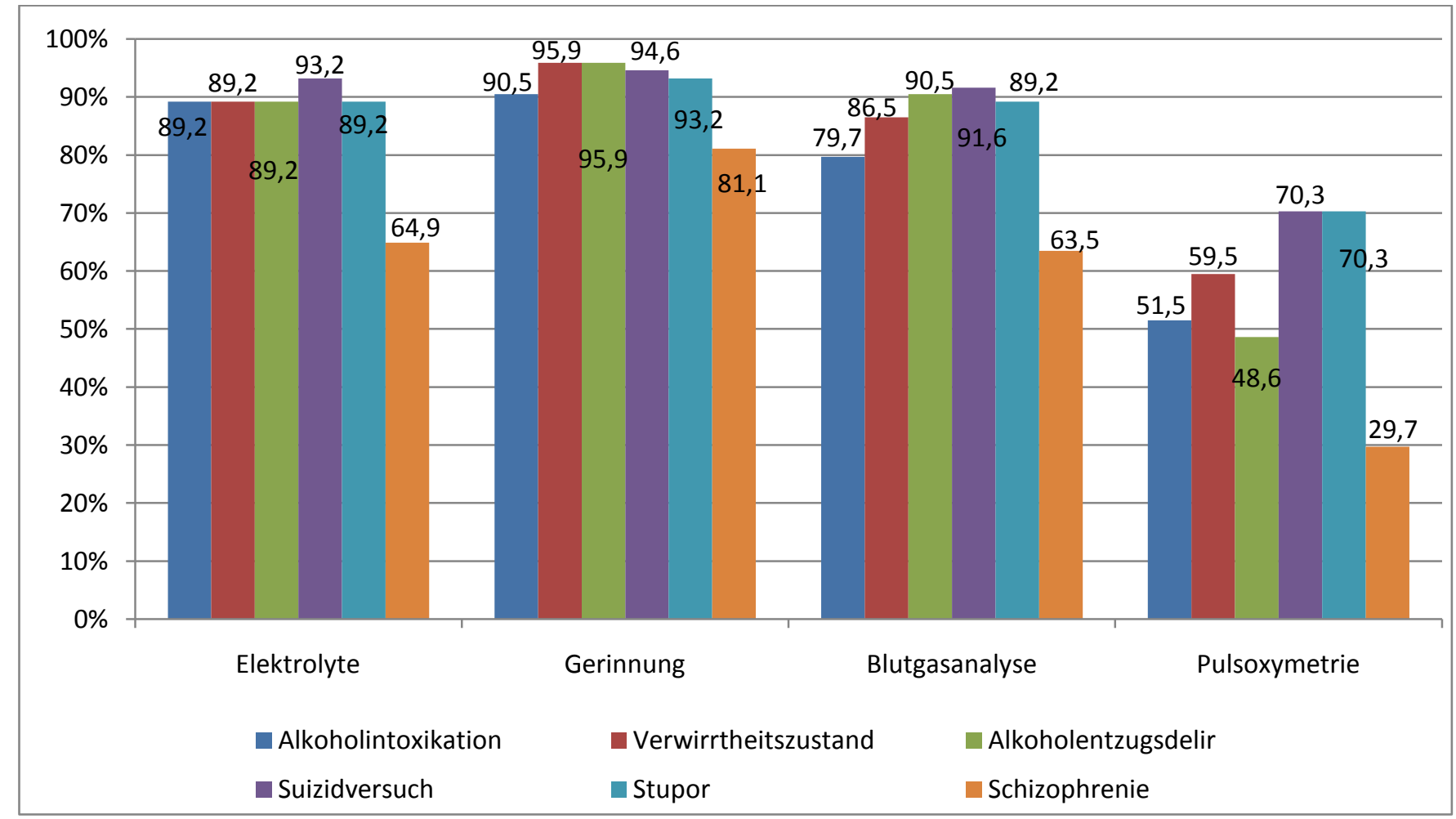

Abbildung 4.8: Versorgung von psychiatrischen Notfallsituationen in der Notaufnahme (4) 


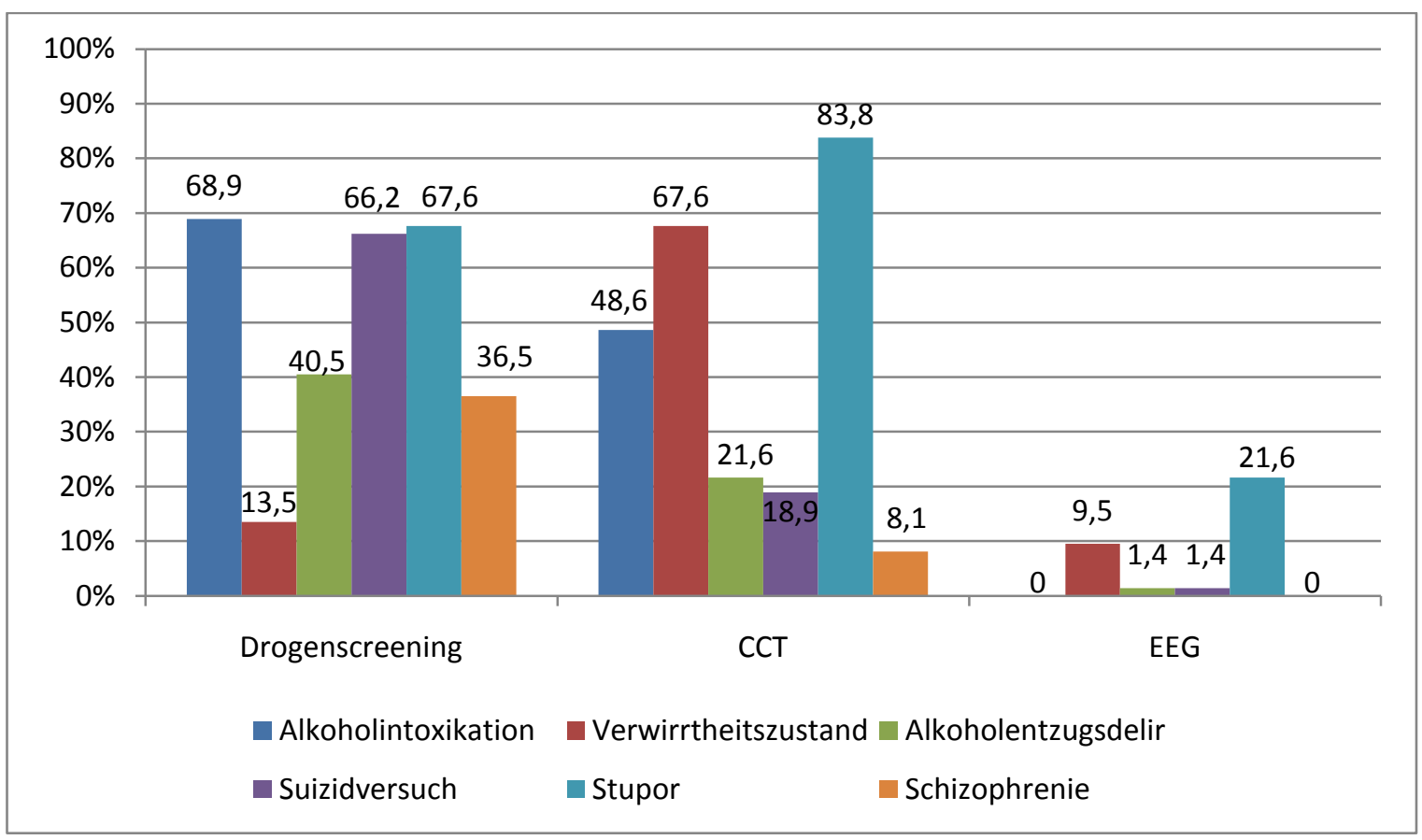

Abbildung 4.9: Versorgung von psychiatrischen Notfallsituationen in der Notaufnahme (5) 


\section{Diskussion}

\subsection{Literaturübersicht}

\subsubsection{Notfallmedizin und Notaufnahme}

Die (zentrale) Notaufnahme eines Krankenhauses ist die primäre Anlaufstelle zur Akutversorgung von Patienten und stellt neben der prästationären Notfallbehandlung einen wichtigen Bestandteil der Notfallmedizin dar. Die (zentrale) Notaufnahme ist nicht nur die Schnittstelle zwischen der Versorgung eines Patienten durch einen Notarzt am Notfallort und dem stationären Bereich eines Krankenhauses [56], denn Patienten werden auch primär in einer Notaufnahme vorstellig. In einer Notaufnahme werden in kurzer Zeit wichtige diagnostische und therapeutische Entscheidungen gefällt, die den Behandlungsverlauf eines akut Erkrankten maßgeblich beeinflussen und deren richtige Einschätzung und Durchführung möglicherweise lebensrettend sind.

Die Durchführung lebensrettender Maßnahmen, das Herstellen der Transportfähigkeit und die Transportbegleitung bei Notfallpatienten sowie der Beginn und die Bahnung diagnostischer und therapeutischer Handlungen stellen die Hauptaufgaben der Notfallmedizin dar, die auch gesetzlich verankert sind. Diese verantwortungsvolle Aufgabenstellung spiegelt sich auch in der Ausbildung, Handlungsweise und fachlichen Kompetenz der in der Notfallmedizin bzw. im Rettungsdienst tätigen Ärzte wieder [34].

Als schwierig stellt sich immer wieder die Abgrenzung eines Notfalls mit vitaler Bedrohung zu einer klinischen Behandlungsnotwendigkeit in der Notaufnahme dar. In der präklinischen Notfallmedizin (Notarzt, Rettungsdienst, kassenärztlicher Notdienst) werden die Einsatzkräfte immer wieder mit Situationen konfrontiert, die ihrem Indikationsspektrum nicht entsprechen - dies jedoch auch bei nicht psychiatrischen Notfallsituationen. Die Notaufnahme als Schnittstelleninstitution ist dagegen Sammelbecken der präklinischen Notfallmedizin, von Polizei und Feuerwehr sowie aller Patienten, die selbstständig vorstellig werden. Während es in der präklinischen Notfallmedizin Richtlinien dafür gibt, welche Versorgungsstruktur Patienten mit welchen Störungen behandeln soll (als hilfreich erscheint hier folgende Definition, die im Rettungsdienstgesetz des Landes Baden-Württemberg verankert ist [1]: Notfallpatienten sind „Kranke oder Verletzte, die sich in einem 
lebensbedrohlichen Zustand befinden oder bei denen schwere gesundheitliche Schäden zu befürchten sind, wenn sie nicht umgehend medizinische Hilfe erhalten"), müssen Notaufnahmen alle Patienten versorgen, gleich wie akut oder lebensbedrohlich diese Erkrankungen sind.

\subsubsection{Der psychiatrische Notfall}

Bei einem psychiatrischen Notfall handelt es sich um ein Ereignis oder einen Zustand, der durch eine psychische Störung oder Krankheit bedingt ist und einer sofortigen Diagnostik und/oder Therapie bedarf, um eine Gefährdung des Betroffenen oder anderer Personen abzuwenden [52]. Eine gelungene Zusammenfassung des ärztlich-therapeutischen Vorgehens bei psychiatrischen Notfällen wurde kürzlich im Deutschen Ärzteblatt veröffentlicht [36].

Für den Notarztdienst in Deutschland liegen mittlerweile einige gute Untersuchungen zur Häufigkeit, zum Patientenspektrum und zur Versorgung psychiatrischer Notfälle vor [20, 22, 23, 24, 34, 37, 40, 41, 51, 55, 57, 67]. Aus Notaufnahmen in Deutschland gibt es bislang nur eine methodisch gut durchgeführte Untersuchung [28]. Daher werden die folgenden Aussagen aus den Untersuchungen im Notarztdienst übernommen.

Leitsymptome des psychiatrischen Notfalles sind Störungen des Bewusstseins, des Antriebs und der Stimmung, wobei in der Regel mindestens zwei dieser Domänen beeinträchtigt sind [44]. Folgende Syndrome werden zu den wichtigsten und häufigsten Akutsituationen im Rahmen eines psychiatrischen Notfalles gezählt [53]:

- akute Suizidalität und selbstschädigendes Verhalten

- Intoxikationen

- Erregungszustände

- Angststörungen

- akute Psychosen

- Delirien und Verwirrtheitszustände

- katatone Syndrome

Bei den meisten psychiatrischen Notfällen liegen chronische oder rezidivierende psychische Erkrankungen vor, vor allem exogene (z.B. Intoxikationen), organische 
(z.B. Verwirrtheitszustand bei Demenz) oder endogene (z.B. schizophrene Psychosen) Psychosen. Häufig führen auch Krisen zu einem psychiatrischen Notfall. Diese entwickeln sich zum psychiatrischen Notfall, wenn ein Ungleichgewicht zwischen der subjektiven Bedeutung eines Problems, der individuellen Resilienz und den zu Verfügung stehenden Bewältigungsstrategien vorliegt, die erlernten Strategien zur Lösung von Problemen („coping behavior“) können das entstandene Lebensproblem nicht bewältigen. Gerade der zunehmende Verlust sozialer, familiärer und gesellschaftlicher Bindungen des einzelnen Individuums in unserer heutigen Zeit, gepaart mit der abnehmenden Bereitschaft und auch Fähigkeit zum Bearbeiten und Bewältigen von Krisen, führt häufiger zur Auslösung psychiatrischer Erkrankungen (z.B. in Form von Belastungsstörungen oder Angsterkrankungen) und auch zu Suiziden bzw. Suizidversuchen [39]. Von Weber, Laux, Luiz und Pajonk wurden vier typische Populationen definiert, die oft psychiatrischer Hilfe im Rahmen der Notfallmedizin bedürfen [44, 46, 75, 76]:

- Chronisch psychisch Kranke, bei denen es durch psychosoziale Belastungsfaktoren zu Exazerbation oder Rezidiven kommt

- Personen mit akuten psychiatrischen Krankheitsbildern

- Personen in akuten psychosozialen Krisensituationen im Gefolge von schwer belastenden, lebensverändernden Ereignissen, oft Verlustereignissen

- Somatisch Kranke mit sekundärer psychiatrischer Störung

- Alte Menschen

Hat man früher - eingedenk der Indikationsliste für den Einsatz des Notarztdienstes - den Eindruck vermittelt bekommen, dass psychiatrische Notfälle so gut wie gar nicht vorkamen [13], so haben die Untersuchungen aus den letzten Jahren zu einer deutlichen Änderung dieser Auffassung geführt. Die Häufigkeit psychiatrischer Notfälle im Notarztdienst in Deutschland beträgt $10-15 \%$ [44]. Darüber hinaus sind nach Einschätzung von Notärzten und Rettungssanitätern bei ungefähr 30 - 40\% aller Notfälle psychologisches Wissen und psychologische Vorgehensweise notwendig [9]. Dem gegenüber steht, dass nur $24 \%$ der Notärzte ihre psychiatrischen Kenntnisse als gut einschätzen [43].

Nach Kropp et al. waren in der von inm untersuchten zentralen Notaufnahme der Medizinischen Hochschule Hannover 7,7\% psychiatrische Patienten. Die häufigsten 
psychiatrischen Diagnosen waren demnach Alkoholintoxikation (20,2\%), paranoide Schizophrenie (14,2\%) und akute Belastungsreaktionen (6,7\%). Er wies jedoch darauf hin, dass von allen psychiatrischen Patienten der Notaufnahme nur 7,8\% durch einen Feuerwehr - oder Polizeieinsatz - darin enthalten ist der Notarzt- und Rettungsdienst - eingeliefert wurden, 56,8\% stellten sich auf Eigeninitiative vor, 25,9\% auf Betreiben Dritter (z.B. Angehörige, Ärzte, Sozialarbeiter) [28]. Die mittlere Behandlungsdauer in der ZNA betrug $123 \pm 97$ (Median: 95) Minuten [27]. Über 50\% der Patienten wurden stationär aufgenommen, vor allem wegen Demenz und Suizidalität [80]. Alkoholassoziierte Erkrankungen fanden sich bei 30,7\% [65]. Kropp et al. nennen weitere Details aus ihrer Untersuchung [28]:

- geringe psychiatrische Fachkompetenz

- $\quad$ keine standardisierten Diagnostikinstrumente

- keine Therapiestandards

Tonn et al. stellten dagegen in einer retrospektiven Untersuchung an einem Krankenhaus der Regelversorgung fest, dass nahezu 32\% aller Patienten, die durch Einsatz eines Notarztes der zentralen Notaufnahme zugeführt wurden, unter einer primären psychiatrischen Erkrankung oder einer psychiatrischen Koerkrankung litten [67], in einer anderen Untersuchung sogar 58\% [66].

In der internationalen Literatur wird davon ausgegangen, dass bis zu $15 \%$ der Patienten in allgemeinen, interdisziplinären Notaufnahmen psychische Störungen aufweisen [72]. In einer Untersuchung in 187 überwiegend ländlichen USamerikanischen Notaufnahmen waren 9,4\% der Patienten psychisch gestört [15]. Bei Verwendung standardisierter Diagnoseinstrumente fanden Marchesi et al., dass sogar $47 \%$ der Patienten einer interdisziplinären Notaufnahme und 54\% der Patienten in einer medizinischen Notaufnahme die Kriterien einer psychischen Störung erfüllten. Die häufigste Diagnose in der interdisziplinären Notaufnahme war die Angststörung (18\%), in der medizinischen Notaufnahme die Depression (21\%) [35]. Circa 36\% der psychiatrischen Patienten sind „Wiederholungsbesucher“. Vorherige stationäre Aufnahmen wegen Depression oder Substanzmissbrauch waren prädiktiv für einen Wiederholungsbesuch [5]. Ungefähr $45 \%$ der Patienten werden aufgenommen, circa 35\% ambulant weiter behandelt, circa $10 \%$ verweigern 
jede Behandlung und ca. 10\% suchen Krisendienste auf [4]. Patienten mit psychiatrischer Komorbidität werden durchschnittlich etwa 5 mal häufiger aufgenommen als Patienten mit nur einer psychiatrischen Diagnose, Patienten mit Psychosen und mit Suizidalität häufiger als Patienten mit anderen Diagnosen [29, 25]. Biancosino et al. untersuchten die Inanspruchnahme des psychiatrischen Konsildienstes in einer großen Notaufnahme über 3 Jahre [3]. Nur 1,4\% der Patienten erhielten ein psychiatrisches Konsil. Von diesen Patienten hatten über Dreiviertel zuvor mindestens einen psychiatrischen Kontakt, über die Hälfte mindestens eine stationäre Aufnahme oder irgendeine Form psychologischer Betreuung. Die häufigsten Diagnosen nach ICD-10 waren neurotische oder Belastungsstörungen (28\%), Schizophrenien (28\%) und Persönlichkeitsstörungen (22\%). Eine US-amerikanische Studie aus Notaufnahmen stellte fest, dass bei psychotischen Patienten zu häufig eine „primäre“ Psychose und zu selten eine substanzinduzierte Psychose diagnostiziert wurde, so hatten 25\% der Patienten mit der Diagnose „primäre“ Psychose eine substanzinduzierte oder keine Psychose [49]. Patienten mit der Diagnose „primäre“ Psychose wurden signifikant häufiger hospitalisiert und mit Antipsychotika behandelt und viel zu selten wurden bei substanzinduzierten Psychosen eine suchtspezifische Weiterbehandlung empfohlen oder eingeleitet [49]. Carpenter et al. wiesen in ihrer Untersuchung darauf hin, dass die Erwartungen der Patienten an die Hilfsangebote einer Notaufnahme oft unrealistisch sind [6].

Psychiatrische Notfallpatienten können auch in einer psychiatrischen Notfallambulanz vorstellig werden. Diese sind jedoch nicht an jeder psychiatrischen Klinik vorhanden. Bislang ist auch unklar, wie viele psychiatrische Notfallambulanzen es in Deutschland gibt. Erst in einer Untersuchung wurde die Versorgungsrealität einer psychiatrischen Notfallambulanz thematisiert. Danach waren die häufigsten Krankheitsbilder, die dort festgestellt wurden, Depressionen, Angststörungen und Anpassungsstörungen (75\%) [47]. Damit unterscheidet sich das Spektrum der Patienten deutlich von dem, welches in allgemeinen Notaufnahmen vorstellig wird.

Psychiatrische Notfälle gehören damit zum Alltag in Notaufnahmen auch deutscher Krankenhäuser. Wenn aber die Vitalfunktionen (Kreislauf, Atmung, Bewusstsein) psychiatrischer Notfallpatienten nicht bedroht sind, wird dieses Patientenklientel häufig nicht angemessen beachtet bzw. erfährt nicht die notwendige Diagnostik und 
Therapie. Dies lässt sich besonders deutlich an Patienten nach Suizidversuch darstellen. Bereits vor 20 Jahren stellten Suokas und Lönnqvist [63] fest, dass 46\% der Patienten, die sich nach einem Suizidversuch in einer Notaufnahme vorstellten, keine psychiatrische Diagnostik erhielten. Die Bedeutung eines Suizidversuchs wurde vom Personal der Notaufnahme generell unterschätzt [62]. In einer Studie von Claassen et al. berichteten in einer Untersuchung an 1590 konsekutiven Patienten von $11,6 \%$ Patienten mit Suizidgedanken und $2 \%$ sogar konkreten Suizidplänen [7]. Suizidalität wurde gravierend unterschätzt. Ältere Patienten wurden häufiger, Frauen und Patienten mit regelmäßiger Beschäftigung wurden seltener aufgenommen. Etwa $50 \%$ der Patienten wurden nach Suizidversuch ohne weitere Nachbehandlung entlassen, entscheidend hierfür waren Alter, psychotische Störung, fehlender Substanzmissbrauch und am meisten das behandelnde Krankenhaus [17, 64].

Dabei machte bereits die Untersuchung von Hickey et al. [18] deutlich, dass Patienten, die im Rahmen eines Index - Suizidversuchs eine psychiatrische Intervention erhielten, nach einem Jahr nur halb so häufig sich erneut selbst schädigten als die, die keine Intervention beim Index - Suizidversuch erhielten. In einer aktuellen Untersuchung aus dem Jahr 2010 stellten die Autoren dar, dass von 286 Suizidtoten sich $124 \mathrm{im}$ Jahr zuvor in einer Notaufnahme vorgestellt hatten, 35 sogar mindestens dreimal [8]. Daher werden zur Identifizierung von Suizidalität auch in Notaufnahmen kurze diagnostische Instrumente empfohlen wie z.B. die Suicide Assessment Scale [74]. Aber auch und gerade diese Patientengruppe braucht in der Notfallambulanz fachkundige und kompetente Hilfe [42].

Auch spielen psychosomatische und psychische Symptome bei chronisch Erkrankten mit einer internistischen Grunderkrankung, die durch diese Grunderkrankung zum Teil vital bedroht sind, eine nicht unbedeutende Rolle [66]. Die Wahrscheinlichkeit, dass sich hinter einer akut aufgetretenen psychischen Störung eine medizinische Ursache verbirgt, wird allerdings in der Literatur extrem unterschiedlich beurteilt und schwankt je nach Untersuchung zwischen 2,5 und 63\% [16, 19, 80]. Darüber hinaus spielen auch so genannte "medically unexplained physical symptoms (MUPS) eine wichtige Rolle [60]. Darunter werden in der Literatur körperliche Symptome ohne relevanten organmedizinischen Befund verstanden. Bei diesen ist eine frühzeitige Ab- und Aufklärung unter Berücksichtigung psychosozialer Faktoren erforderlich. Ein solches Vorgehen verhindert nicht notwendige Untersuchungen und Aufnahmen und 
vermindert eine Verstärkung, die Wiedervorstellungen und falsche Coping Mechanismen begünstigen könnte.

Daher ist bei jedem psychisch kranken oder auffälligen Patient eine vollständige körperlich - neurologische Untersuchung, unter Einschluss von Laboruntersuchungen und gegebenenfalls apparativer Diagnostik unverzichtbar. Die notwendige somatomedizinische Abklärung psychisch kranker Menschen wird als Medical Clearance bezeichnet.

Durch eine gründliche Anamnese und klinische Untersuchung kann ein Großteil der Patienten bezüglich der Beschwerdeursachen richtig zugeordnet werden. Die Sensitivität, medizinische Ursachen allein aufgrund der gründlichen Anamnese herauszufinden, betrug in einer Untersuchung 94\%. Durch eine gründliche klinische Untersuchung konnte immerhin eine Sensitivität von 51\% erreicht werden [26].

Für die Durchführung einer Laboruntersuchung wird in der Literatur eine Sensitivität von 20\% für die Diagnosefindung angegeben [38]. In Verbindung mit der Anamnese und Klinik sind Laborwerte aber ein wertvoller Baustein. Wie umfassend das Notfallscreening aussehen sollte, wird jedoch kritisch diskutiert. Blutbild, Kreatinin, Kreatininkinase, Glukose und Elektrolyte (Natrium, Kalium, Calcium) gehören zum Standard [16, 38, 69, 78]. Es bleibt immer zu bedenken, dass klinisch irrelevante Laborveränderungen in die Irre führen können [16].

Uneinigkeit besteht in der Literatur bei der Frage, ob ein Drogen- und Alkoholscreening generell durchgeführt werden sollte. Viele Autoren empfehlen ein Screening. Schiller et al. [50] fanden jedoch heraus, dass nur 10\% der Patienten vom Arzt nicht als Drogenkonsumenten erkannt wurden und dabei den Konsum nicht selbst zugaben. Die Weiterbehandlung der Patienten war zudem vom Ergebnis eines routinemäßig durchgeführten Urin- und Drogenscreenings unabhängig und erhöhte die Kosten der Behandlung ohne erkennbaren Mehrwert. Auch Olshaker et al. [38] berichteten, dass die Patientenangaben bezüglich illegaler Drogen zu über $90 \%$ verlässlich waren.

Die meisten Autoren messen der Anamnese kombiniert mit der körperlichen Untersuchung den größten Stellenwert zu. Weiterführende Untersuchungen wie z.B. Röntgenthorax oder Lumbalpunktion sollten nur gezielt bei begründetem Verdacht 
eingesetzt werden, da sie keinen wesentlichen Informationsgewinn liefern und stattdessen die Patienten verunsichern und die Untersuchungskosten in die Höhe treiben [16]. Nur Henneman et. al. [16] empfehlen, bei jedem Patienten eine CCTUntersuchung und eine Lumbalpunktion durchzuführen. Bei dieser Studie muss jedoch bemerkt werden, dass es sich um ein vorselektiertes Patientengut handelte, da Patienten mit offensichtlicher Drogen- oder Alkoholintoxikation, psychiatrische Patienten mit nebenbefundlichen medizinischen Problemen, psychiatrische Patienten mit kurz zuvor diagnostizierten Verhaltensauffälligkeiten und akut suizidale Patienten ausgeschlossen wurden.

Bemerkenswert ist, dass es im diagnostischen Vorgehen eine Diskrepanz zwischen notfallmäßig aufnehmenden Psychiatern und Notfallmedizinern gibt. Im Umgang mit Notfallsituationen erfahrene und entsprechend ausgebildete somatische Ärzte verlassen sich offensichtlich eher auf Anamnese und klinische Untersuchung, dahingehend, dass sie nur wenige Routinelabortests veranlassen. In einer Studie von Zun et al. [81] führten Notfallmediziner circa ein bis vier Tests durch, am häufigsten Drogen- und Alkoholscreening sowie ein Blutbild. Psychiater in dieser Situation forderten im Schnitt fünf bis acht Tests, wobei auch hier Drogen- und Alkoholscreening sowie Blutbild am häufigsten durchgeführt wurden. Auch insgesamt war die Art der Zusatzuntersuchungen ähnlich. Die Psychiater verursachten zwei- bis dreimal so hohe Kosten wie die Somatiker. Es wurde vermutet, dass die Notfallmediziner Tests eher bedarfsorientiert einsetzen, Psychiater routinemäßig eher etwas breiter gefächert. In der Summe der Routinetests und zusätzlich zur Routine georderten Untersuchungen sowie der Art der Tests ergaben sich wieder Übereinstimmungen. Psychiatern könnten damit Unsicherheiten in der somatischen Versorgung unterstellt werden, Somatikern dagegen die Neigung, psychisch auffällige Patienten schnell zu verlegen und zu schnell als "gefiltert" zu kategorisieren.

\subsubsection{Psychiatrisches Management von psychiatrischen Notfällen}

Wie bereits dargelegt, werden in Notaufnahmen psychiatrische, insbesondere akute psychiatrische Erkrankungen selten wahrgenommen. Insbesondere in großen Kliniken ist es notwendig, dass ein Psychiater fest einer zentralen Notaufnahme 
zugeordnet ist. In der Regel der Fälle ist die Erhebung eines primären psychischen bzw. psychiatrischen Befundes Aufgabe des diensthabenden, nicht psychiatrischen Arztes, deshalb muss der diensthabende Arzt in der Lage sein, einen psychischen Befund eindeutig erheben um daraus ein Entscheidung hinsichtlich der weiteren Behandlung einleiten zu können.

Bei viele Patientinnen und Patienten ist eine psychiatrische Erkrankung leicht zu diagnostizieren, wie zum Beispiel Entzugs- oder Intoxikationssymptome bei Suchtpatienten oder psychotische Patienten im Zustand nach einer selbstaggressiven Handlung. Andererseits gibt es viele Patienten, die oft nur mit unspezifischen und somatischen Symptomen in einer Notaufnahme vorstellig werden und deren psychiatrische Grunderkrankung nicht auf den ersten Blick auffällt [54, 79].

Der auch in Notaufnahmen zu erhebende basale psychische Befund kann in vier Kategorien und weiteren Subkategorien aufgeteilt werden [68]:

- Bewusstsein

- Quantitatives Bewusstsein

- Qualitatives Bewusstsein

- Affektivität

- Stimmung

- Antrieb

- Denk- und Wahrnehmungsleistungen

- Denkleistungen

- Wahrnehmung

- Kognitive Leistungen

- Gedächtnis

- Kognition

Die Erhebung der oben beschriebenen Befundparameter wird wohl in den meisten interdisziplinären Notaufnahmen in unterschiedlichem Umfang durchgeführt.

Jeder Patient in einer Notaufnahme muss quantitativ (z.B. mittels Glasgow - Coma Scale) und qualitativ in seinem Bewusstsein beurteilt werden. Auch gehört die Prüfung der Orientierung (Person, Ort, Zeit und Situation) zu den 
Untersuchungsstandards. Darüber hinaus lassen sich Stimmung und Antrieb bereits im Anamnesegespräch mit dem Patienten qualitativ bestimmen.

Gerade bei Ärztinnen und Ärzten, die keine oder nur geringe psychiatrische Erfahrungen besitzen, bietet sich der Einsatz validierter Fragebögen als weiterführendes Diagnoseinstrumentarium an. Von Spitzer et al. [58] wurde 1994 ein gut und schnell ausführbarer und auswertbarer Fragebogen entwickelt: „primary care evaluation of mental disorders" (PRIME-MD), von dem es eine deutsche Version gibt [32]. In Weiterentwicklung bzw. in Ergänzung zu diesem Fragesatz kann in Deutschland der „Patient's Health Questionnaire“ (PHQ) [58, 12] eingesetzt werden. Der PHQ ist auf seine Diagnosequalität mehrfach mit guten Ergebnissen - zum Beispiel für Depression [33] - untersucht worden. Auch der Einsatz des General Health Questionnaire-30 (GHQ-30) wird empfohlen [35]. Das Mini International Neuropsychiatric Interview (MINI) sowie das Composite International Diagnostic Interview (CIDI) sind für den nicht psychiatrisch geschulten Arzt in der Notaufnahme weniger geeignet, zudem benötigen beide Fragesätze für ein gutes Ergebnis mit 30 bis 60 Minuten einen zu großen Zeitaufwand [71]. Insbesondere bei älteren Patienten empfiehlt sich, um dementielle oder depressive Störungen besser zu erkennen, der ergänzende Gebrauch von vier weiteren Testverfahren: Uhrentest, Mini-MentalStatus-Test, DemTect und die Geriatrische Depressions-Skala (GDS15). Diese Verfahren können in zentralen bzw. interdisziplinären Notaufnahmen gut und schnell durchgeführt werden und liefern hilfreiche diagnostische Aussagen [10, 21, 70, 77].

\subsection{Methodische Fragen}

Bei der hier vorliegenden Untersuchung handelt es sich um eine anonyme Befragung zur Versorgung psychiatrischer Notfälle in (zentralen) Notaufnahmen deutscher Krankenhäuser. Die daraus gewonnenen Ergebnisse unterliegen in ihrer Aussagefähigkeit den typischen Einschränkungen von Erhebungen dieser Art.

Es gingen 74 verwertbare Antworten auf die schriftlichen Anfragen sowie die Aufrufe über Fachgesellschaften und Webseiten ein. Eine Rücklaufrate kann als Folge dieses Verfahrens nicht errechnet werden. Der Rücklauf ist sicher zu gering, als dass die Ergebnisse als repräsentativ für alle Notaufnahmen in Deutschland bezeichnet werden könnten, aber hoch genug, um erste wichtige Aussagen über die Versorgung 
psychiatrischer Notfälle in Notaufnahmen treffen und weitere Hypothesen generieren zu können. Es handelt sich um die erste Befragung deutscher Notaufnahmen zu diesem Thema.

Die Gründe für diese eher niedrige Beteiligung sind vielfältig. Es ist nicht bekannt, wie viele der angeschriebenen Krankenhäuser auch über eine Notaufnahme verfügten. Das Thema wird immer noch von vielen Notaufnahmen als nicht relevant betrachtet. Der Fragebogen ist mit fünfzehn Druckseiten außergewöhnlich umfangreich. Vielfach wurden im Fragebogen Detailangaben gefordert, so dass z.B. Fallzahlen in der Verwaltung oder im Klinikinformationssystem recherchiert werden mussten. Die Bearbeitungszeit lag nach Aussagen einzelner Teilnehmer bei circa 45 Minuten, etwaige Anfragen in der Verwaltung nicht eingeschlossen. Das teilweise unvollständige Ausfüllen gerade von Daten, die aus der Verwaltung zur Verfügung gestellt werden mussten (z.B. Fallzahlen), macht deutlich, dass möglicherweise in manchen Kliniken die Teilnahme an der Befragung als zu aufwendig betrachtet wurde, nicht erwünscht war oder auch verboten wurde. Da die Portokosten für die Rücksendung des Fragebogens von den Antwortenden selber getragen werden mussten, könnte dies die Teilnahme, insbesondere derjenigen, die sich für die Thematik dieser Arbeit nicht interessierten, noch weiter gesenkt haben. Unter diesen Gesichtspunkten ist ein Rücklauf von 74 Fragebögen noch als eher hoch zu bezeichnen.

Die Ergebnisse dieser Arbeit stellen den Meinungsspiegel einer Personengruppe dar, die auf die Befragung antworteten. Es konnte nicht nachgeprüft werden, in wie weit die gemachten Angaben auf Daten eines Klinikinformationssystems beruhen oder willkürlich geschätzt wurden, bzw. bei Schätzungen, ob es sich um eine individuelle Schätzung oder eine im Team konsentierte Schätzung handelt. Durch die Befragungsart, so wurde bei den Fragen nach der Häufigkeit bestimmter psychiatrischer Störungen nach geschätzten Häufigkeiten gefragt, können sowohl Ungenauigkeiten wie auch Verzerrungen, z. B. im Sinne der Erwünschtheit, entstehen, die gerade auch durch die Anonymität der Fragebögen nicht auszuschließen sind.

Auch wurde keine bestimmte Berufsgruppe innerhalb der Notaufnahmen (z.B. Ärzte, Pflegepersonal) direkt zur Mitarbeit angesprochen. Die Briefe wurden an den Leiter 
der Notaufnahme gesendet. Ob dieser die Befragung selbst beantwortete oder jemand anderen damit beauftragte, konnte nicht geprüft werden. Insofern blieb auch der berufliche Erfahrungsstand und die berufliche Qualifikation (z. B. Facharztstatus) des tatsächlich Antwortenden unklar. Bis auf vier Fragebögen (durch Pflegerische Leitungen) wurden aber alle durch Ärzte ausgefüllt.

Sicherlich unterliegen auch Wertungen wie z.B. „sehr schlecht" und „nicht immer ausreichend“ oder die Differenz zwischen „befriedigend“ und "sehr gut", wie sie in der Frage nach der selbstbeurteilten Rechtssicherheit bei Unterbringungsmaßnahmen gewählt wurden, individuellen Unterschieden. Hilfreich und bewährt ist hier eine Unterteilung in gestaffelte Antwortmöglichkeiten in Anlehnung an eine Art Schulnotensystem, wie auch in diesem Erhebungsbogen vollzogen, so dass hier im Verhältnis zum Aufwand eine recht zuverlässige Methode der Bewertung gewählt wurde.

Alle Fragen wurden in etwa gleicher Anzahl beantwortet. Die Rate nicht beantworteter Fragen war bei keiner Frage größer als 6\%. Eine Ausnahme bildet lediglich die Frage nach der Rechtssicherheit, hier machten 17,6\% keine Angabe. Damit können die Fragen insgesamt als verständlich und der Aufbau des Fragebogens als logisch angesehen werden.

Zusammenfassend ist bei der Bewertung dieser Einschätzungen zu berücksichtigen, dass durch eine solche Binnenbefragung der durch die Teilnehmer angegebene Stellenwert psychiatrischer Themen in der Notaufnahme isoliert betrachtet wurde und nicht in Relation zur Meinung gegenüber anderen Bereichen der Notfallmedizin gesetzt wurde. Eine Untersuchung, in der psychiatrische in Beziehung zu anderen notfallmedizinischen Themen beurteilt werden, bleibt somit anderen Arbeiten vorbehalten.

Trotz methodenimmanenter und geringer inhaltlicher Schwäche bleibt festzustellen, dass sich der Erhebungsbogen als verwertbares Instrument erwiesen hat, der gemessen hat, was er messen sollte. Der hohe Anteil der vorausgesagten und tatsächlich eingetroffenen Aussagen und Korrelationen legt nahe, dass die Ergebnisse valide sind. 


\subsection{Diskussion der Ergebnisse}

Die Einsendungen kamen aus Krankenhäusern aller Versorgungsstufen, vielleicht mit leichtem Schwerpunkt aus Häusern der Maximal- und Schwerpunktversorgung. Entsprechend der Versorgungsstufe variierten auch die Anzahl der Krankenhausbetten und die Anzahl der Patienten im Referenzzeitraum eines Jahres in der Notaufnahme. Mit etwa $75 \%$ beteiligten sich vor allem interdisziplinäre Notaufnahmen. In fast allen Notaufnahmen waren die Innere Medizin und die Chirurgie vertreten, insgesamt waren in den antwortenden Notaufnahmen 18 medizinische Disziplinen vertreten. Im Mittel arbeiteten dort vier Ärzte tagsüber, drei Ärzte nachts oder am Wochenende. Zusammen mit der breiten Verteilung über das Bundesgebiet kann von einer Stichprobe ausgegangen werden, die im Wesentlichen typisch für die "Landschaft" der Notaufnahmen in Deutschland ist. Ähnliches gilt für die Antwortenden: Das Durchschnittsalter betrug circa 45 Jahre, etwa zwei Drittel der Antwortenden waren Männer.

In vielen Notaufnahmen fand sich bereits eine psychiatrische Kompetenz, entweder in Form von Fachärzten sowie Ärzten mit mindestens einjähriger Tätigkeit in der Psychiatrie - dies können z.B. auch Fachärzte für Neurologie sein - oder in Form eines psychiatrischen Konsildienstes oder einer psychiatrischen oder psychosomatischen Klinik vor Ort. Bei über $80 \%$ der antwortenden Notaufnahmen war eine psychiatrisch - psychotherapeutische oder psychosomatische Klinik oder ein Konsildienst verfügbar, in gut $30 \%$ ein Facharzt oder Arzt mit mindestens einjähriger Tätigkeit in der Psychiatrie. Nur in 9 Notaufnahmen (12,2\%) fand sich gar keine psychiatrische Kompetenz. Diese 9 Notaufnahmen verteilten sich interessanterweise recht homogen auf alle Versorgungsstufen (Maximalversorgung: $n=2$; Schwerpunktversorgung: $n=2$; Regelversorgung: $n=3$; Grundversorgung: $n=2$ ) und fanden sich auch in interdisziplinären Notaufnahmen $(n=5$; internistische Notaufnahmen: $n=2$; chirurgische Notaufnahmen: $n=1$; keine Angabe: $n=1$ ). Damit liegt - wenn auch auf unterschiedlichem Niveau - eine recht flächendeckende Versorgung psychiatrischer Patienten in deutschen Notaufnahmen durch Facheinrichtungen oder-personal vor.

Allerdings ist es bemerkenswert, dass viele Notaufnahmen nicht genau das Leistungsspektrum psychiatrischer Einrichtungen vor Ort kennen. So war es $46 \%$ der 
Antwortenden nicht bekannt, ob es eine psychiatrische Aufnahmestation, 42\% nicht bekannt, ob es eine psychiatrische Notaufnahme und 7\% nicht bekannt, ob es einen psychiatrischen Konsildienst vor Ort gibt. Im Rahmen dieser Untersuchung konnten die genauen Gründe hierfür nicht geklärt werden. Mögliche Erklärungen könnten darin bestehen, dass diese Notaufnahmen entweder psychiatrische Notfälle als nicht spezifisch behandlungsbedürftig beurteilen oder glauben, diese Notfälle suffizient mit ihren Mitteln behandeln zu können - deshalb auf psychiatrische Notaufnahmen und Aufnahmestationen verzichten zu können.

Die Teilnehmer gaben an, dass circa $15 \%$ der in einem Referenzzeitraum von einem Jahr in deutschen Notaufnahmen vorstelligen Patienten psychisch auffällig waren, circa 9\% sogar psychiatrisch diagnose- und therapiebedürttig. Bei etwa 14\% habe dementsprechend eine psychiatrische Erkrankung bestanden, bei Abzug von Intoxikationen und Suchterkrankungen bei etwa 7\%. Circa 11\% der Patienten sei eine psychopharmakologische Medikation verordnet worden, ein psychiatrisches Konsil sei bei circa $5 \%$ durchgeführt worden. Letzteres bedeutet, dass nach eigener Einschätzung der Antwortenden nur etwa ein Drittel aller psychiatrischen Patienten ein Konsil erhält. Der Anteil der Patienten in Notaufnahmen, die ein psychiatrisches Konsil erhalten, ist aber an Standorten, an denen eine psychiatrische Klinik oder ein psychiatrischer Konsildienst verfügbar sind (5,5\%) nur etwas höher als an Standorten, an denen diese Angebote fehlen (3,3\%). Damit ließe sich die oben geäußerte Argumentation erhärten, dass die meisten deutschen Notaufnahmen der Auffassung sind, die meisten psychiatrischen Notfälle selbst diagnostizieren und therapieren zu können. Auch die Tatsache, dass zwar 15\% der Patienten als psychisch auffällig, aber nur $9 \%$ als diagnose- und therapiebedürftig gewertet wurden, könnte damit ihre Erklärung finden.

Der Anteil psychiatrischer Patienten liegt höher als der von Kropp et al. ermittelte Wert von 7,7\% [28], aber auch deutlich unter dem aus der Arbeitsgruppe von Tonn genannten Wert (32\%) [67]. Er entspricht vielmehr recht genau dem Wert, der in den meisten internationalen Studien genannt wurde (15\%) [72]. Diese Diskrepanzen können z.T. mit erheblichen Unterschieden in der Methodik der Untersuchung erklärt werden. Bei Verwendung standardisierter Skalen ist es zu erwarten, dass der Anteil psychischer Störungen noch erheblich höher, möglicherweise bis nahe $50 \%$ liegen könnte [35]. Leider werden in Deutschland standardisierte Screeningfragebögen oder 
Selbsteinschätzungsskalen zum Feststellen einer psychischen Erkrankung nur sehr selten, in unserer Untersuchung von nur vier Notaufnahmen angewendet.

In unserer Untersuchung stellte sich heraus, dass etwa die Hälfte aller psychischen Störungen in der Notaufnahme auf Suchterkrankungen zurückgeführt wurden (Psychiatrische Erkrankungen einschließlich Suchterkrankungen: 14,2\%; Psychiatrische Erkrankungen exklusive Suchterkrankung: 6,8\%). Auch in anderen Untersuchungen - national und international - war der Anteil der Erkrankungen durch den Einsatz psychotroper Substanzen (Alkohol und Drogen) recht hoch und schwankte zwischen 30\% alkoholassoziierten Erkrankungen [65] bis zu 50\% [5].

Der Anteil von Patienten mit Suizidversuchen und Suizidalität wird möglicherweise erheblich unterschätzt. In unserer Stichprobe wurden durchschnittlich $2 \%$ der Patienten mit Suizidversuchen in Notaufnahmen gesehen. Eine englische Untersuchung von Claassen \& Larkin berichtete ebenfalls über $2 \%$ der Patienten mit konkreten Suizidplänen, die sich wegen nicht psychischer Störungen in einer allgemeinen Notaufnahme vorstellten. Darüber hinaus wurden in dieser Gruppe aber weitere $12 \%$ mit Suizidgedanken festgestellt [7]. Dies wird bestätigt durch eine Untersuchung aus dem deutschen Notarztdienst, in der explizit nach Suizidalität gefragt wurde, auch wenn diese nicht primär zu vermuten gewesen wäre. Hier fanden sich ebenfalls und überraschend $7-9 \%$ der Patienten mit Suizidgedanken [46]. In unserer Untersuchung wurde lediglich nach bereits erfolgten Suizidversuchen gefragt, daher ist zu vermuten, dass darüber hinaus mindestens weitere $10 \%$ der Patienten Suizidpläne oder -gedanken haben.

Aggressive Patienten spielen eine wichtige Rolle in Notaufnahmen. Auch wenn sie selten sind, so können sie viel Schaden anrichten und die notwendige sachliche Überschau in einer Notaufnahme durch hohe Emotionalität behindern. In unserer Untersuchung wurden durchschnittlich 3\% der Patienten als aggressiv angegeben, im Mittel wurden 2,5 Mitarbeiter durch aggressive Patienten im Jahr verletzt. Uns sind keine Vergleichszahlen aus anderen Untersuchungen bekannt. Aus dem Rettungsdienst ist aber berichtet worden, dass es vermehrt Übergriffe gegen Personen und Gegenstände gibt. Innerhalb eines Jahres waren circa zwei Drittel der Rettungsdienstmitarbeiter Opfer von Gewalt geworden, teilweise mehr als viermal. 
15\% der untersuchten Rettungsdienstmitarbeiter erlitten eine körperliche Schädigung [31].

Der Umgang sowohl mit suizidalen als auch mit aggressiven Patienten stellt für Notaufnahmen unverändert ein großes Problem dar. Insbesondere ist es vielen Mitarbeitern nicht klar, unter welchen Bedingungen sie Maßnahmen gegen den Willen des Patienten bei Vorliegen einer psychischen Erkrankung einleiten können und müssen. Die Rechtssicherheit bei diesen Fragen wurde von über $40 \%$ als nicht ausreichend beurteilt, weitere knapp 20\% machten hierzu keine Aussage. 15\% der Notaufnahmen berichteten gar, keine gerichtlichen Unterbringungen durchzuführen, und in knapp 50\% seien gerichtliche Unterbringungen nur mit Unterstützung durch einen Psychiater vorgenommen worden. Dabei sind gerichtliche Unterbringungen nach Aussagen der Teilnehmer keine Rarität (im Mittel 50 pro Jahr), sie werden somit circa 1 Mal pro Woche durchgeführt.

Als wichtigste diagnostische Verfahren in der Notaufnahme wurden die körperliche Untersuchung (97\%), die Bestimmung von Blutdruck und Pulsfrequenz (97\%) sowie die Durchführung eines EKG (77\%) genannt, bei den Laboruntersuchungen die Bestimmung von Glukose (93\%), Blutbild (88\%) und Elektrolyten (87\%). Bildgebende Verfahren wurden nur selten als Standarddiagnostikverfahren genannt (Röntgen Thorax: 16\%, CCT: 26\%). Nur in etwa einem Drittel aller Notaufnahmen gehörte ein Drogenscreening zum Standardprocedere der Diagnostik. Am seltensten wurden die Elektroenzephalographie (1\%) und die Blutgasanalyse (16\%) genannt. Damit halten sich die Notaufnahmen im Wesentlichen an die Empfehlungen aus einschlägigen Publikationen. Hinsichtlich der Laborbestimmungen wird jedoch empfohlen, bei allen psychiatrischen Patienten zumindest Blutbild, Kreatinin, Kreatininkinase, Glukose und Elektrolyte durchzuführen. Bildgebende Verfahren sollten nur gezielt bei begründetem Verdacht eingesetzt werden [16, 38, 69, 78]. Hinsichtlich eines Drogenscreenings kann darauf verwiesen werden, dass nach genauer Anamneseerhebung und Untersuchung nur bei etwa $10 \%$ der Patienten ein tatsächlicher, durch Screeningverfahren bestätigter Alkohol- oder Drogenkonsum durch klinische Verfahren nicht festgestellt wurde [38, 50]. Damit erscheint ein routinemäßig durchgeführtes Drogenscreening in Notaufnahmen nicht sinnvoll. 
Bei $11 \%$ aller Patienten in der Notaufnahme, so wurde geschätzt, wurde eine psychopharmakologische Behandlung durchgeführt. Dies ist ein relevanter Anteil, der vermuten lässt, dass Psychopharmaka auch gekannt und entsprechend ihrer Indikation eingesetzt werden. Bei genauer Betrachtung der Ergebnisse erscheint dies jedoch zweifelhaft.

In fast allen Notaufnahmen waren Lorazepam und Diazepam oral verfügbar, in zwei Drittel Haloperidol und Promethazin. Parenteral wurden Diazepam und Haloperidol in $90 \%$ aller Notaufnahmen als verfügbar angegeben. Verblüffend waren die Angaben zu Oxazepam und Pipamperon. Hier fragten wir als Kontrolle auch die Verfügbarkeit der parenteralen Form ab, obwohl es diese in Deutschland gar nicht gibt. So gaben 6,8\% der Teilnehmer an Oxazepam, 10,8\% Pipamperon in parenteraler Form in der Notaufnahme vorrätig zu haben.

Als unverzichtbar wurden von etwa 90\% der Notaufnahmen vor allem Diazepam, Lorazepam und Haloperidol klassifiziert. Knapp 50\% beurteilten Promethazin als unverzichtbar. Alle anderen genannten Medikamente (Levomepromazin, Amitryptilin, Oxazepam, Melperon, Pipamperon) wurden nur zu einem geringen Teil benannt. Die gängigen Publikationen zur Behandlung psychiatrischer Notfälle mit Empfehlungscharakter bestätigen die Notwendigkeit des Vorhandenseins von Lorazepam, Diazepam, Haloperidol und Melperon. Letzteres ist besonders zur Therapie von Unruhezuständen bei älteren Menschen geeignet, da es keine anticholinergen Eigenschaften aufweist. Aus psychiatrischer Sicht wird dem Levomepromazin der Vorzug vor dem Promethazin gegeben [45, 68]. Amitryptilin ist als Notfallmedikament sicher nicht geeignet, selbst wenn $10 \%$ der teilnehmenden Notaufnahmen dies als unverzichtbar bezeichneten.

Haloperidol wurde von $77 \%$ als parenteral unverzichtbar eingeschätzt. Dieser Anteil mag im letzten Jahr weiter zurückgegangen sein. Im September 2007 warnte die Arzneimittelkommission der Deutschen Ärzteschaft vor dem intravenösen Einsatz von Haloperidol. In 28 Fällen war es weltweit zum Auftreten maligner Herzrhythmusstörungen als Folge einer QTc-Zeit Verlängerung, zum Teil mit Todesfolge, gekommen [11]. Im Jahr 2009 entschloss sich der Hersteller, die Firma Janssen-Cilag, Haloperidol parenteral nur noch zur intramuskulären Gabe zu empfehlen. Diese Empfehlung basierte laut Pressemitteilung nicht auf neuen Daten, 
sondern auf einer geänderten Einschätzung der bestehenden Datenlage. So seien die in der Fachinformation empfohlenen Sicherheitsvorkehrungen (kontinuierliches EKG-Monitoring unter i.v. Applikation) nicht eingehalten worden.

Angesichts der Ergebnisse zur Beurteilung von Psychopharmaka bleiben doch einige ernste Zweifel, ob die teilnehmenden Notaufnahmen tatsächlich über die Verfügbarkeit und die Indikationsstellung der wichtigsten Psychopharmaka in hinreichenden Maß informiert sind. Letztlich bleiben auch Zweifel an der korrekten Ausfüllung des Fragebogens, wenn Medikamente als „vorhanden“ bezeichnet werden, die es in dieser Darreichungsform gar nicht gibt. Vielmehr scheint es, als gäbe es in diesem Punkt einen erheblichen Fortbildungsbedarf, auch um die Auswahl der Psychopharmaka in Notaufnahmen zu begrenzen und auf evidenzbasierte Kriterien zurückzuführen.

In Abhängigkeit davon, ob eine psychiatrische oder psychosomatische Klinik am Standort vorhanden ist, ergaben sich in der Beantwortung des Fragebogens keine größeren Unterschiede. Notaufnahmen an Standorten mit psychiatrischer oder psychosomatischer Klinik berichteten lediglich über signifikant weniger Suizidversuche, eine höhere Anzahl gerichtlicher Unterbringungen und eine höhere Anzahl verletzter Mitarbeiter. Dies könnte damit erklärt werden, dass suizidale Patienten möglicherweise direkt in die psychiatrische Klinik eingeliefert werden. Die höhere Anzahl gerichtlicher Unterbringungen und aggressiver Patienten mag durch die Besonderheit der Notaufnahme mit psychiatrischer Klinik am Standort bedingt sein, möglicherweise wird diese Notaufnahme durch Notarzt und Rettungsdienst bei „schwierigen“ Patienten bevorzugt angefahren.

Keine relevanten Unterschiede ergaben sich auch in Abhängigkeit von der Frage nach dem Vorhandensein psychiatrischer Kompetenz am Standort. Bei der Frage nach der Beurteilung der Unverzichtbarkeit von Psychopharmaka ergaben sich lediglich einige grenzwertige Unterschiede. An Standorten, an denen eine psychiatrische oder psychosomatische Klinik vorhanden ist, wurde Lorazepam eher für unverzichtbar gehalten. Dies galt ähnlich für Standorte mit psychiatrischer Kompetenz, an diesen Standorten wurde Amitryptilin tendenziell eher für verzichtbar gehalten. 
Männer und Frauen beantworteten die Fragen zur Häufigkeit und Therapie psychiatrischer Störungen in den meisten Fällen signifikant unterschiedlich, in dem Sinne, dass Teilnehmerinnen durchweg höhere Prozentzahlen angaben. So berichteten Frauen von circa 18\% psychisch Auffälligen in Notaufnahmen, Männer dagegen nur von circa 14\%. Erklärungen aus der Literatur gibt es hierfür nicht. Es lässt sich lediglich vermuten, dass Frauen hinsichtlich psychischer Störungen in der Wahrnehmung sensibler sind. In den Fragen, die besser operationalisierbar und konkretisierbar waren (z.B. Suizidversuche, Anteil Psychopharmakotherapie) fanden sich keine geschlechtsspezifischen Unterschiede.

Ebenso gab es signifikante Unterschiede bei den Fragen nach der Häufigkeit psychisch auffälliger Patienten und von Suizidversuchen in der Notaufnahme in Abhängigkeit von der Art der Notaufnahme. So wurden psychisch Auffällige und Suizidversuche deutlich häufiger in rein internistischen und interdisziplinären Notaufnahme genannt als in rein chirurgischen Notaufnahmen. Psychisch auffällige und suizidgefährdete Patienten werden primär wohl besser in interdisziplinären oder internistischen Notaufnahmen versorgt als in rein Chirurgischen. Die Mehrzahl aller Suizidversuche erfolgt durch Intoxikationen (z.B. Alkohol, Drogen, Medikamente, Gase). Suizidversuche mit der Folge eines Traumas sind seltener und enden häufiger tödlich, daher werden Suizidversuche in ausschließlich chirurgischen Notaufnahmen sicher seltener gesehen. Beim Vergleich der Versorgungsstufen der Krankenhäuser fand sich lediglich ein Unterschied bei der Häufigkeit psychiatrischer Erkrankungen exklusive Suchterkrankungen, diese wurde in Krankenhäusern der Regelversorgung (10\%) deutlich häufiger als in Krankenhäusern der Maximalversorgung (4\%) angegeben.

Die Fallbeispiele wurden insgesamt differenziert bearbeitet. Bis auf die schizophrene Patientin wären alle anderen Patienten überwiegend somatisch aufgenommen worden. Auch in Notaufnahmen, an deren Standort sich eine psychiatrische Klinik befindet, war diese Einschätzung nicht wesentlich anders. In diesen Notaufnahmen wäre lediglich der delirante Alkoholabhängige und der stuporöse Patient präferenziell in die Psychiatrie aufgenommen worden.

Ein psychiatrisches Konsil beim Alkoholintoxikierten wäre nur von einem knappen Viertel der Notaufnahmen durchgeführt worden. Dabei wäre bei der Mehrzahl dieser 
Patienten eine Motivation zur Reduktion des Konsums, die Feststellung eines Missbrauchs oder einer Abhängigkeit und die dann erforderliche Aufklärung über die Strukturen des Suchthilfesytems durch qualifiziertes Personal nicht nur wünschenswert, dadurch könnte auch die Aufsuchfrequenz der Notaufnahme durch diese Patienten gesenkt werden.

Die Standardverfahren zur Diagnostik wurden bei allen Fallbeispielen als erforderlich betrachtet, mit einer kleinen Einschränkung bei der schizophrenen Patientin: diese hätte in circa $10 \%$ der Notaufnahmen keine körperliche Untersuchung und keine Bestimmung von Blutdruck und Pulsfrequenz erhalten, in 34\% der Notaufnahmen kein EKG, in $15-35 \%$ keine angemessene Untersuchung von Laborparametern. Dies erscheint nicht gerechtfertigt, auch bei einer akuten Exazerbation einer schizophrenen Psychose müssen diese Verfahren komplett durchgeführt werden. Dabei sind vor allem Herzrhytmussstörungen, Blutbildveränderungen, Veränderungen der Elektrolyte und der Hepatozytenfunktion als Folge der unregelmäßig eingenommenen Medikation auszuschließen.

Spezialuntersuchungen wurden differenziert eingesetzt. Die Durchführung einer Röntgenaufnahme des Thorax wurde vor allem zum Ausschluss organischer Ursachen indiziert und in den Fallbeispielen der Alkoholintoxikation und der Schizophrenie nur selten „angeordnet“. Ein Drogenscreening ist von etwa zwei Drittel der teilnehmenden Notaufnahmen beim alkoholintoxikierten Patienten, bei der Patientin mit Zustand nach Suizidversuch und beim stuporösen Patienten als notwendig erachtet worden. Ein CCT wurde vor allem beim stuporösen Patient und beim akuten Verwirrtheitszustand für erforderlich gehalten. Das EEG ist in aller Regel als Notfallmaßnahmen nur im Status epilepticus sinnvoll. Beim stuporösen Patienten ist dies eine der möglichen Differenzialdiagnosen.

Damit kann festgestellt werden, dass die Teilnehmer bis zum Ende des Fragebogens engagiert, aufmerksam und konsequent mitgearbeitet haben. Die psychiatrische Versorgung in Notaufnahmen deutscher Krankenhäuser ist besser als erwartet. In den meisten Notaufnahmen ist eine psychiatrische Kompetenz durch Mitarbeiter in der Notaufnahme oder psychiatrische Institutionen vor Ort gewährleistet. Vielfach bestehen jedoch in den Notaufnahmen zu wenig Informationen oder Unkenntnis über das Leistungsangebot der psychiatrischen Institutionen. Konsilangebote, wenn sie 
vorhanden sind, werden eher zu selten genutzt, vor allem auch bei Suchterkrankungen. Der Anteil psychiatrischer Erkrankungen ist mit 15\% hoch relevant; möglicherweise ist er aber, nach Verwendung geeigneter Diagnoseinstrumente noch höher. Ebenfalls unterschätzt werden dürfte der Anteil suizidaler Patienten. Die Anzahl intoxikierter Patienten, die Alkohol oder Drogen missbräuchlich oder im Rahmen einer Abhängigkeit einnehmen und sich damit in einer Notaufnahme vorstellen, ist erkennbar hoch. Die Frage, in wie weit Notaufnahmen in diesem Rahmen nicht Aufgaben in der Prävention übernehmen können, sollte offen diskutiert werden. Es kann von einer zufriedenstellend hohen diagnostischen Sicherheit bei eher geringen (psychopharmako-) therapeutischen Kenntnissen ausgegangen werden. Eine große Unsicherheit findet sich auch in der rechtlichen Beurteilung bei Patienten, bei denen Maßnahmen gegen deren Willen getroffen werden sollen oder müssen. Der größte Fortbildungsbedarf ist sicher in den rechtlichen Grundlagen und in der Pharmakotherapie psychiatrischer Notfälle zu sehen. 


\section{Literaturverzeichnis}

1. Baden-Württemberg R 97. Gesetz über den Rettungsdienst (Rettungsdienstgesetz-RDG) vom 19. November 1991 (GBI. S. 713); geändert durch Gesetz vom 18. Dezember 1995 (GBI. S. 879) Hrsg Sozialministerium Baden-Württemberg

2. Berardi D, Berti Ceroni G, Leggieri G, Rucci P, Ustün B, Ferrari G (1999) Mental, physical and functional status in primary care attenders. Int $J$ Psychiatry Med 29: 133-148

3. Biancosino B, Vanni A, Marmai L, Zotos S, Peron L, Marangoni C, Magri V, Grassi $L$ (2009) Factors related to admission of psychiatric patients to medical wards from the general hospital emergency department: a 3-year study of urgent psychiatric consultations. Int J Psychiatry 39: 133-146

4. Bruffaerts R, Sabbe M, Demyttenaere K (2005) Predicting aftercare in psychiatric emergencies. Soc Psychiatry Psychiatr Epidemiol 40: 829-834

5. Bruffaerts R, Sabbe M, Demyttenaere K (2006) Who visits the psychiatric emergency room for the first time? Soc Psychiatry Psychiatr Epidemiol 41: 580-586

6. Carpenter LL, Schecter JM, Underwood JA, Tyrka AR, Price LH (2005) Service expectations and clinical characteristics of patients receiving psychiatric emergency services. Psychiatr Serv 56: 743-745

7. Claassen CA, Larkin GL (2005) Occult suicidality in an emergency department population. Br J Psychiatry 186: 352-353

8. Da Cruz D, Pearson A, Saini P, Miles C, While D, Swinson N, Williams A, Shaw, J, Appleby L, Kapur N (2010) Emergency department contact prior to suicide in mental health patients. Emerg Med $J$, epub ahead of print

9. D`Amelio R, Archonti C, Falkai P, Pajonk FG (2006) Psychologische Konzepte und Möglichkeiten der Krisenintervention in der Notfallmedizin. Notfall Rettungsmed 9: 194-204 
10. D'Ath P, Katona P, Mullan E, Evans S, Katona C (1994) Screening, detection and management of depression in elderly primary care attenders. I: The acceptability and performance of the 15 item Geriatric Depression Scale (GDS15) and the development of short versions. Fam Pract 11: 260-266

11. Deutsches Ärzteblatt (2007) Tödliche kardiale Risiken von Haloperidol. http://www.aerzteblatt.de/nachrichten/29858/

12. Diez-Quevedo C, Rangil T, Sanchez-Planell L, Kroenke K, Spitzer RL (2001) Validation and utility of the patient health questionnaire in diagnosing mental disorders in $\mathbf{1 0 0 3}$ general hospital Spanish inpatients. Psychosom Med 63: 679-686

13. Ellinger K (1998) Psychiatrische Notfälle im Notarztdienst. In Hewer W und Rössler W (Hrsg.), Das Notfall Psychiatrie Buch. Urban \& Schwarzenberg: 535-536

14. Griffies WS, Collins WJ, Adams AE (2007) The Louisiana emergency department behavioral health challenge. J La State Med Soc 159: 268-275

15. Hartley D, Ziller EC, Loux SL, Gale JA, Lambert D, Yousefian AE (2007) Use of critical access hospital emergency rooms by patients with mental health symptoms. J Rural Health 23: 108-115

16. Henneman PL, Mendoza R , Lewis RJ (1994) Prospective evaluation of emergency department medical clearance. Ann Emerg Med 24: 672-677

17. Hepp U, Moergeli H, Trier SN, Milos G, Schnyder U (2004) Attempted suicide: factors leading to hospitalization. Can J Psychiatry 49: 736-742

18. Hickey L, Hawton K, Fagg J, Weitzel H (2001) Deliberate self-harm patients who leave the accident and emergency department without a psychiatric assessment. A neglected population at risk of suicide. J Psychosom Res 50: $87-93$

19. Janofsky JS, Tamburello AC (2006) Diversion to the mental health system: emergency psychiatric evaluations. J Am Acad Psychiatry Law 34: 283-291 
20. Kaff A (1987) Aufgaben und Zielsetzung der Wiener Rettung. Intensivmed Notfallmed 24: 94-98

21. Kalbe E, Kessler J, Calabrese P, Smith R, Passmore AP, Brand M, Bullock R (2004) DemTect: a new, sensitive cognitive screening test to support the diagnosis of mild cognitive impairment and early dementia. Int J Geriatr Psychiatry 19: 136-143

22. Kanz KG, Neumann M (1997) Einsatzanalyse eines großstädtischen Notarztdienstes als Grundlage für Bedarfs- und Qualitätsplanung im Rettungsdienst. Intensivmed Notfallmed 34: 85

23. Kardels B, Beine KH (2003) Psychiatrische Notfälle in Hamm/Westfalen. Fortschr Neurol Psychiatr 71: 129-134

24. König F, König E, Wolfersdorf M (1996) Zur Häufigkeit des psychiatrischen Notfalls im Notarztdienst. Notarzt 12: 12-17

25. Kolbasovsky A, Reich L, Futterman R, Meyerkopf N (2007) Reducing the number of emergency department visits and costs associated with anxiety: a randomized controlled study. Am J Manag Care 13: 95-102

26. Korn CS, Currier GW, Henderson SO (2000) "Medical clearance" of psychiatric patients without medical complaints in the emergency department. J Emerg Med 18: 173-176

27. Kropp S, Andreis C, te Wildt B, Reulbach U, Ohlmeier M, Auffarth I, Ziegenbein M (2005) Psychiatric patients turnaround times in the emergency department. Clin Pract Epidemiol Ment Health 1: 27

28. Kropp S, Andreis C, te Wildt B, Sieberer M, Ziegenbein M, Huber TJ (2007) Charakteristik psychiatrischer Patienten in der Notaufnahme. Psychiatr Praxis 34: 72-75 
29. Kunen S, Prejean C, Gladney B, Harper D, Mandry CV (2006) Disposition of emergency department patients with psychiatric comorbidity: results from the 2004 National Hospital Ambulatory Medical Care Survey. Emerg Med J 23: 274-275

30. Lecrubier $Y(2007)$ Widespread underrecognition and undertreatment of anxiety and mood disorders: results from 3 European studies. J Clin Psychiatry 68: 36-41

31. Lenk M (2008) Aggressionsverhalten gegenüber Mitarbeitern der Notfallrettung. Bachelor-Thesis zur Erlangung des akademischen Grades Bachelor of science in public health and administration, Hochschule Neubrandenburg, Fachbereich Gesundheit und Pflege, Studiengang Gesundheitswissenschaften

32 Loerch B, Szegedi A, Kohnen R, Benkert O (2000) The primary care evaluation of mental disorders (PRIME-MD), German version: a comparison with the CIDI. J Psychiatr Res 34: 211-220

33. Löwe B, Spitzer RL, Gräfe K, Kroenke K, Quenter A, Zipfel S, Buchholz C, Witte S, Herzog W (2004) Comparative validity of three screening questionnaires for DSM-IV depressive disorders and physicians' diagnoses. J Affect Disord 78: 131-140

34. Luiz T, Huber T, Schieth B, Madler C (2000) Einsatzrealität eines städtischen Notarztdienstes: Medizinisches Spektrum und lokale Einsatzverteilung. Anästhesiologie und Intensivmedizin 41: 765-773

35. Marchesi C, Brusamonti E, Borghi C, Giannini A, di Ruvo R, Minneo F, Quarantelli C, Maggini C (2004) Anxiety and depressive disorders in an emergency department ward of a general hospital: a control study. Emerg Med J 21: 175-179

36. Mavrogiorgou P, Brüne M, Juckel G (2011) Ärztlich-therapeutisches Vorgehen bei psychiatrischen Notfällen. Deutsches Ärzteblatt 13: 222-229 
37. Obertacke U, Wissing $H$ (1987) Der Stellenwert des Notarztrettungswesen in der Großstadt Essen - Erfahrungen der ersten zehn Jahre.

Notfallmedizin 13: 186-208

38. Olshaker JS, Browne B, Jerrard DA, Prendergast H, Stair TO (1997) Medical clearance and screening of psychiatric patients in the emergency department. Acad Emerg Med 4: 124-128

39. Pajonk FG, Poloczek S, Schmitt TK (2000) Der psychiatrische Notfall. Notfall Rettungsmed 3: 363-370

40. Pajonk FG, Bartels HH, Biberthaler P, Bregenzer T, Moecke H (2001) Der psychiatrische Notfall im Rettungsdienst - Häufigkeit, Versorgung und Beurteilung durch Notärzte und Rettungsdienstpersonal. Nervenarzt 72 : 685-692

41. Pajonk FG, Grünberg K (2001) Psychiatrische Notfälle im Notarztdienst einer deutschen Großstadt. Fortschr Neurol Psychiatr 69: 170-174

42. Pajonk FG, Moecke HP (2002) Psychiatrie im Rettungsdienst. Notfall Rettungsme 5: 87-88

43. Pajonk FG, Lubda J, Sittinger H, Moecke H, Andresen B, von Knobelsdorff G (2004) Psychiatrische Notfälle aus der Sicht von Notärzten. Anaesthesist 8: $709-716$

44. Pajonk FG, Stöwer S, Kinn M, Fleiter B (2006) Psychopharmakotherapie in der Notfallmedizin. Notfall Rettungsmed 9: 393-402

45. Pajonk FG, Schmitt P, Biedler A, Richter JC, Meyer W, Luiz T, Madler C (2008) Psychiatric emergencies in prehospital emergency medical systems: a prospective comparison of two urban settings. Gen Hosp Psychiatry 30: 360-366

46. Pajonk FG, Moecke $H(2009)$ Psychiatrische Notfälle in der Notfallmedizin - Definition, Häufigkeit, Epidemiologie. In: Madler C, Jauch KW, Werdan K, Siegrist J, Pajonk FG (Hrsg) Akutmedizin - Die ersten 24 Stunden. Das NAWBuch 4. Aufl. Elsevier, München: 729-733 
47. Quandt F, Wendel S, Degirmenci Ü, Kreil S, Bayerlein K, Rotter A, Kornhuber J, Weith M (2011) Der Patient in der psychiatrischen Notambulanz: Erstdiagnosen, Vorstellungsgründe und Vergleich der Laien- vs. Arztsicht. Fortschr Neurol Psychiatr 79: 221-225

48. Rothschild AJ, Winer J, Flint AJ, Mulsant, Whyte EM, Heo M, Fratoni S, Gabriele M, Kasapinovic S, Meyers BS (2008) Missed diagnosis of psychotic depression at 4 academic medical centers. J Clin Psychiatry 69: 1293-1296

49. Schanzer BM, First MB, Dominguez B, Hasin DS, Caton CL (2006) Diagnosing psychotic disorders in the emergency department in the context of substance use. Psychiatr Serv 57: 1468-1473

50. Schiller MJ, Shumway M, Batki SL (2000) Utility of routine drug screening in a psychiatric emergency setting. Psychiatr Serv 51: 474-478

51. Schmidt-Matthiesen A, Kreuzer J (1993) Spektrum und Effektivität der Notfallversorgung durch Chirurgen als Notwagenärzte. Zentrbl Chir 118 : $69-75$

52. Schmitt TK, Pajonk FG, Poloczek S (2000) Psychiatrische Notfälle und Krisen. Notfall Rettungsmed 3: 531-538

53. Schönfeldt-Lecuona C, Dirks B, Wolf RC, Pajonk FG, Freudenmann RW, Höse A, Connemann BJ (2008) Psychiatrische Notfälle im Notfall- und Rettungswesen. Notfall Rettungsmed 11: 525-530

54. Schriger DL, Gibbons PS, Langone CA, Lee S, Altshuler LL (2001) Enabling the diagnosis of occult psychiatric illness in the emergency department: a randomized, controlled trial of the computerized, self-administered PRIME-MD diagnostic system. Ann Emerg Med 37: 132-140

55. Sefrin P (2003) Der Notarztdienst als interdisziplinäre Aufgabe. Notfallmedizin 29: 529-538 
56. Sefrin P (2010) Schnittstelle Notarztdienst und Notaufnahme (Interface between emergency physician service und emergency room). Der Notarzt 26: $256-259$

57. Seiger K, Brommundt J (2002) Krisenintervention im Rettungsdienst eine Herausforderung an den Notarzt? Notfall Rettungsmed 5: 116-118

58. Spitzer RL, Williams JB, Kroenke K, Hornyak R, McMurray J (2000) Validity and utility of the PRIME-MD patient health questionnaire in assessment of $\mathbf{3 0 0 0}$ obstetric-gynecologic patients: the PRIME-MD Patient Health Questionnaire Obstetrics-Gynecology Study. Am J Obstet Gynecol 183: 759-769

59. Stebbins LA, Hardman GL (1993) A survey of psychiatric consultations at a suburban emergency room. Gen Hosp Psychiatry 15: 234-242

60. Stephenson DT, Price JR (2006) Medically unexplained physical symptoms in emergency medicine. Emerg Med J 23: 595-600

61. Summers WK, Rund DA, Levin M (1979) Psychiatric illness in a general urban emergency room: daytime versus nighttime population. $J$ Clin Psychiatry 40: 340-343

62. Suokas J, Lönnqvist J (1989) Evaluation of attempted suicides: A comparative study of staff in a general hospital and consulting staff in a psychiatric hospital. Crisis 10: 123-131

63. Suokas J, Lönnqvist J (1991) Selection of patients who attempted suicide for psychiatric consultation. Acta Psychiatr Scand 83: 179-182

64. Suominen KH, Isometsä ET, Lönnqvist JK (2004) Attempted suicide and psychiatric consultation. Eur Psychiatry 19: 140-145

65. te Wildt B, Andreis C, Auffarth I, Tettenborn C, Kropp S, OhImeier M (2006) Alcohol related conditions represent a major psychiatric problem in emergency departments. Emerg Med J 23: 428-430 
66. Tonn P, Reuter S (2005) Hilflose Patienten-hilflose Retter? Psychiatrische Notfälle im Rettungsdienst. Rettungsdienst 11: 1094-1099

67. Tonn P, Gerlach N, Reuter S, Friedrich B, Dahmen N (2006) Häufigkeit von psychiatrischen Diagnosen bei Patienten in der notärztlichen Versorgung: Eine retrospektive Studie. Intensivmedizin 43: 123-129

68. Tonn P, Reuter S, Gerlach N, Dahmen N, Pajonk FG (2008) Psychiatrische Patienten in der Notaufnahme. Notfall Rettungsmed 537-546

69. Tueth MJ (1994) Diagnosing psychiatric emergencies in the elderly. Am J Emerg Med 12: 364-369

70. van der Cammen TJ, van Harskamp F, Stronks DL, Passchier J, Schudel WJ (1992) Value of the Mini-Mental State Examination and informants' data for the detection of dementia in geriatric outpatients. Psychol Rep 71: 1003-1009

71. van Vliet IM, de Beurs E (2007) The MINI-International Neuropsychiatric Interview. A brief structured diagnostic psychiatric interview for DSM-IV en ICD-10 psychiatric disorders. Tijdschr Psychiatr 49: 393-397

72. Villari V, Rocca P, Bogetto F (2007) Emergency psychiatry. Minerva Med 98 : 525- 541

73. Vitale SG, van de Mheen D, Garretsen HF, van de Wiel A (2005) Selfreported alcohol use among Dutch emergency room patients: variations in prevalence rates owing to methodological differences. Alcohol Alcohol 40: $524-530$

74. Waern M, Sjöström N, Marlow, T, Hetta J (2010) Does the Suicide Assessment Scale predict risk of repetition? A prospective study of suicide attempters at a hospital emergency department. Eur Psychiatry 25: $421-426$

75. Weber M, Laux T, Luiz T, Pajonk FG (2005) Psychiatrische Erkrankungeneine Notarztindikation? Ein Vergleich mit anderen Einsatzkategorien. Deutscher Anästhesie Kongress (DAC), München 
76. Weber M, Madler C, Pajonk FG (2005) Zur Problematik psychiatrischer Notarzteinsätze. Notfall Rettungsmed 8: 489-492

77. Weyerer S, Eifflaender-Gorfer S, Köhler L, Jessen F, Maier W, Fuchs A, Pentzek M, Kaduszkiewicz H, Bachmann C, Angermeyer MC, Luppa M, Wiese $B$, Mösch E, Bickel $H$ (2008) Prevalence and risk factors for depression in non-demented primary care attenders aged 75 years and older. J Affect Disord 111: 153-163

78. Williams ER, Shepherd SM (2000) Medical clearance of psychiatric patients. Emerg Med Clin North Am 18: 185-198

79. Wulsin LR, Hillard JR, Geier P, Hissa D, Rouan GW (1988) Screening emergency room patients with atypical chest pain for depression and panic disorder. Int J Psychiatry Med 18: 315-323

80. Ziegenbein M, Anreis C, Brüggen B, Ohlmeier M, Kropp S (2006) Possible criteria for inpatient psychiatric admissions: which patients are transferred from emergency services to inpatient psychiatric treatment? BMC Health Serv Res 6: 150

81. Zun LS, Hernandez R, Thompson R, Downey L (2004) Comparison of EPs' and psychiatrists' laboratory assessment of psychiatric patients. Am J Emerg Med 22: 175-180 


\section{Anhang}

\subsection{Fragebogen}

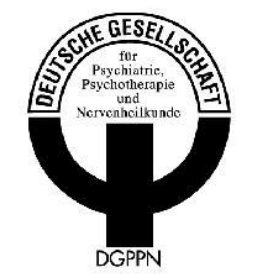

Referat Notfallpsychiatrie

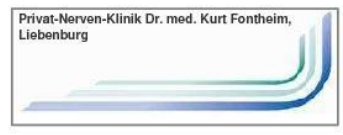

Arbeitsgruppe Psychiatrie und Notfallmedizin

Wissenschaftliche Untersuchung

\section{Psychiatrische Versorgung in der Notaufnahme}

Sehr geehrte Frau Kollegin,

sehr geehrter Herr Kollege,

Patienten mit psychischen Störungen finden sich häufig in der Notaufnahme. Nach einer Untersuchung von Kropp et al. (2006) handelt es sich um mindestens 7,7\% in einer interdisziplinären Notaufnahme eines Großklinikums. Es wird jedoch davon ausgegangen, dass viele psychische Störungen in der Notaufnahme nicht erkannt werden. In Notaufnahmen ohne unmittelbar erreichbare psychiatrische Klinik wird die Versorgung psychisch Kranker sicher anders aussehen als in Kliniken mit psychiatrischer Klinik oder Abteilung.

Eine systematische Erfassung der Häufigkeit psychischer Störungen in Notaufnahmen und deren Diagnostik- und Behandlungsmöglichkeiten ist bislang nicht erfolgt. Das Referat „Notfallpsychiatrie“ der Deutschen Gesellschaft für Psychiatrie, Psychotherapie und Nervenheilkunde (DGPPN) und die Arbeitsgruppe „Psychiatrie und Notfallmedizin“ (AGPUN) bitten Sie daher recht herzlich, den folgenden Fragebogen auszufüllen. Ziel dieser wissenschaftlichen Untersuchung ist es, den Versorgungsbedarf besser einzuschätzen, um daraus Standards für Diagnostik und Therapie entwickeln zu können.

Mit Ihrer Teilnahme stimmen Sie auch der wissenschaftlichen Verwertung der Daten in Form von Publikationen zu, wobei selbstverständlich die Anonymität vollständig gewahrt bleibt. In gewohnter Form werden wir allen Teilnehmern eine Auswertung der Ergebnisse zuschicken und Sie über daraus entstehende Publikationen informieren.

Vielen Dank für Ihre Unterstützung!

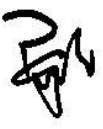

Prof. Dr. med. Frank-Gerald Pajonk

Bitte schicken Sie den ausgefülten Fragebogen, möglichst bis zum 31.05.2009, an:

Prof. Dr. Frank-Gerald Pajonk

Privat-Nerven-Klinik Dr. med. Kurt Fontheim

Lindenstr. 15, 38704 Liebenburg

Email: agpun@klinik-dr-fontheim.de 
Zu Beginn benötigen wir einige wenige Daten über Sie, die/der den Bogen ausfüllt:

Alter: Jahre

Geschlecht $\quad \square \mathrm{m} \quad \square$ w

Sie sind Fachärztin/Facharzt für
$\square \quad$ Allgemeinmedizin
$\square \quad$ Anästhesie
$\square \quad$ Chirurgie
$\square \quad$ Innere Medizin
$\square \quad$ Sonstige

Bitte geben Sie die ersten beiden Stellen der Postleitzahl des Ortes an, in dem sich Ihre Notaufnahme befindet

Die Notaufnahme, auf die sich alle folgenden Angaben beziehen sollen, ist
$\square \quad$ interdisziplinär
$\square \quad$ internistisch
$\square \quad$ chirurgisch
$\square$ sonstige:

Welcher Versorgungsstufe gehört das Krankenhaus an, zu dem die Notaufnahme gehört?
$\square \quad$ Grundversorgung
$\square \quad$ Regelversorgung
$\square \quad$ Schwerpunktversorgung
$\square \quad$ Maximalversorgung

Über wie viele Krankenhausbetten verfügt dieses Klinikum/Krankenhaus? 
Betten

Wie viele Patienten werden in dieser Notaufnahme im Jahr behandelt (nach Möglichkeit als Berechnungsgrundlage das Jahr 2007 annehmen)?

\section{Patienten}

Ärzte welcher Fachrichtungen sind in dieser Notaufnahme im Dienstplan regulär zum Dienst eingeteilt (keine Konsilärzte, Mehrfachnennungen möglich)?

$\begin{array}{llll}\square & \text { Allgemeinmedizin } & \square & \text { Innere Medizin } \\ \square & \text { Anästhesie } & \square & \text { Urologie } \\ \square & \text { Augenheilkunde } & \square & \text { Gynäkologie } \\ \square & \text { Allgemeinchirurgie } & \square & \text { HNO } \\ \square & \text { Unfallchirurgie } & \square & \text { Radiologie } \\ \square & \text { Orthopädie } & \square & \text { Neurologie } \\ \square & \text { Psychiatrie } & \square & \text { Sonstige: }\end{array}$

Wie viele Ärztinnen und Ärzte sind in der Notaufnahme üblicherweise im Dienst?

- In der Regelarbeitszeit (z.B. 8.00 - 16.30 Uhr):

- außerhalb der Regelarbeitszeit:

Wie viele Ärztinnen und Ärzte, die fest in der Notaufnahme arbeiten (eine Konsilärzte),

- sind Fachärztin/Fachart für Psychiatrie (und Psychotherapie) oder Nervenheilkunde?

- haben für mindestens ein Jahr in einer Klinik für Psychiatrie und Psychotherapie gearbeitet? 
Gibt es am Klinikum/Krankenhaus, zu dem diese Notaufnahme gehört, eine

- psychiatrische Klinik/Abteilung $\quad \square$ ja $\quad \square$ nein $\quad \square$ weiß nicht

- psychosomatische Klinik/Abteilung $\square$ ja $\quad \square$ nein $\quad \square$ weiß nicht

- neurologische Klinik/Abteilung $\square$ ja $\quad \square$ nein $\quad \square$ weiß nicht

- einen psychiatrischen Konsildienst

$\square$ nein

$\square$ ja, 24 h verfügbar

$\square$ ja nur innerhalb üblicher Arbeitszeiten (z.B. Mo-Fr 8.00-16.30 Uhr)

$\square$ weiß nicht

Wenn das Klinikum/Krankenhaus, zu dem Ihre Notaufnahme gehört, eine psychiatrische Klinik/Abteilung hat, verfügt diese über eine eigene
Notaufnahme
$\square$ ja
$\square$ nein
$\square$ weiß nicht
(Not-)Aufnahmestation
$\square$ ja $\quad \square$ nein
$\square$ weiß nicht 
Im Folgenden fragen wir Sie nach geschätzten Häufigkeiten von psychischen Auffälligkeiten und psychiatrischen Notfällen in der Notaufnahme. Bitte geben Sie diese in Prozent (\%) bezogen auf alle Notfälle eines Referenzzeitraumes von 1 Jahr (nach Möglichkeit des Jahres 2007) an.

Wir fragen zunächst nach allgemeineren Angaben und werden danach immer spezifischer, so dass Ihre Angaben in der Regel (Ausnahmen sind möglich) von höheren zu geringeren Prozentzahlen abfallen. Auch wenn sich die Prozentangaben nicht aufeinander beziehen, so sollten sie doch in Relation zueinander ein zutreffendes Gesamtbild abgeben. Sollten Sie ungefähre Angaben machen, so setzen Sie bitte ein "ca. "vor die Zahl.

Wie viel Prozent aller Ihrer Patientinnen und Patienten innerhalb eines Referenzzeitraums von 1 Jahr sind psychisch auffällig?

\section{$\%$}

Wie viel Prozent aller Ihrer Patientinnen und Patienten innerhalb eines Referenzzeitraums von 1 Jahr halten Sie für psychiatrisch diagnose- und/oder therapiebedürftig?

$\%$

Wie viel Prozent aller Ihrer Patientinnen und Patienten innerhalb eines Referenzzeitraums von 1 Jahr leidet nach Ihrer Auffassung an einer psychiatrischen Erkrankung (incl. Suchterkrankungen)?

$\%$

Wie viel Prozent aller Ihrer Patientinnen und Patienten innerhalb eines Referenzzeitraums von 1 Jahr leidet nach Ihrer Auffassung an einer psychiatrischen Erkrankung (ohne Suchterkrankungen)?

$\%$

Wie viel Prozent aller Ihrer Patientinnen und Patienten innerhalb eines Referenzzeitraums von 1 Jahr erhalten ein psychiatrisches Konsil?

$\%$

Wie viel Prozent aller Ihrer Patientinnen und Patienten innerhalb eines Referenzzeitraums von 1 Jahr erhalten im weitesten Sinne eine psychopharmakologische Medikation?

$\%$ 
Wie viel Prozent aller Ihrer Patientinnen und Patienten innerhalb eines Referenzzeitraums von 1 Jahr wurden wegen eines Suizidversuchs versorgt?

$\%$

Wie viel Prozent aller Ihrer Patientinnen und Patienten innerhalb eines Referenzzeitraums von $1 \mathrm{Jahr}$ sind handgreiflich/aggressiv geworden?

$\%$

Wie viele Ihrer Mitarbeiterinnen und Mitarbeiter sind innerhalb eines Referenzzeitraums von 1 Jahr durch aggressive Handlungen von Patientinnen oder Patienten verletzt worden?

Leiten Sie in der Notaufnahme gerichtliche Unterbringungen nach Unterbringungsgesetz (UG), Psychisch Kranken Gesetz (Psych KG) oder Bürgerlichem Gesetzbuch (BGB) ein?

nein, keine

$\square \quad$ ja, aber nur mit Unterstützung durch einen Psychiater/Nervenarzt

$\square \quad$ ja, auch ohne Unterstützung durch einen Psychiater/Nervenarzt

wenn ja: wieviele Unterbringungen leiten Sie innerhalb eines Referenzzeitraums von 1 Jahr in der Notaufnahme etwa ein?

Wie schätzen Sie Ihre Rechtssicherheit ein, wenn Sie gerichtliche Unterbringungen nach Unterbringungsgesetz (UG), Psychisch Kranken Gesetz (Psych KG) oder Bürgerlichem Gesetzbuch (BGB) ohne Hilfe eines Psychiaters/Nervenarzt einleiten müssten? (Dies beinhaltet u.a. die Kenntnisse der entsprechenden Gesetze und deren Ausführungsbestimmungen sowie die praktische Umsetzung einer Unterbringung.)
$\square \quad$ sehr schlecht
$\square \quad$ nicht immer ausreichend
$\square \quad$ befriedigend
$\square \quad$ sehr gut 
Werden in der Notaufnahme standardisierte Screeningfragebögen oder Selbsteinschätzungsskalen zum Feststellen einer psychischen Erkrankung bzw. deren Schwere angewendet?

$\square \quad$ nein, keine

$\square \quad$ ja, nämlich
$\square \quad$ PRIME-MD
$\square \quad$ Patient Heath-Questionnaire (PHQ), deutsche Version
$\square \quad$ Minimental State Examination (MMSE)
$\square \quad$ sonstige:

Welche somatischen Untersuchungsverfahren wenden Sie bei psychisch auffälligen Patienten standardmäßig an, selbst wenn keine klare organische Ursache erkennbar ist?

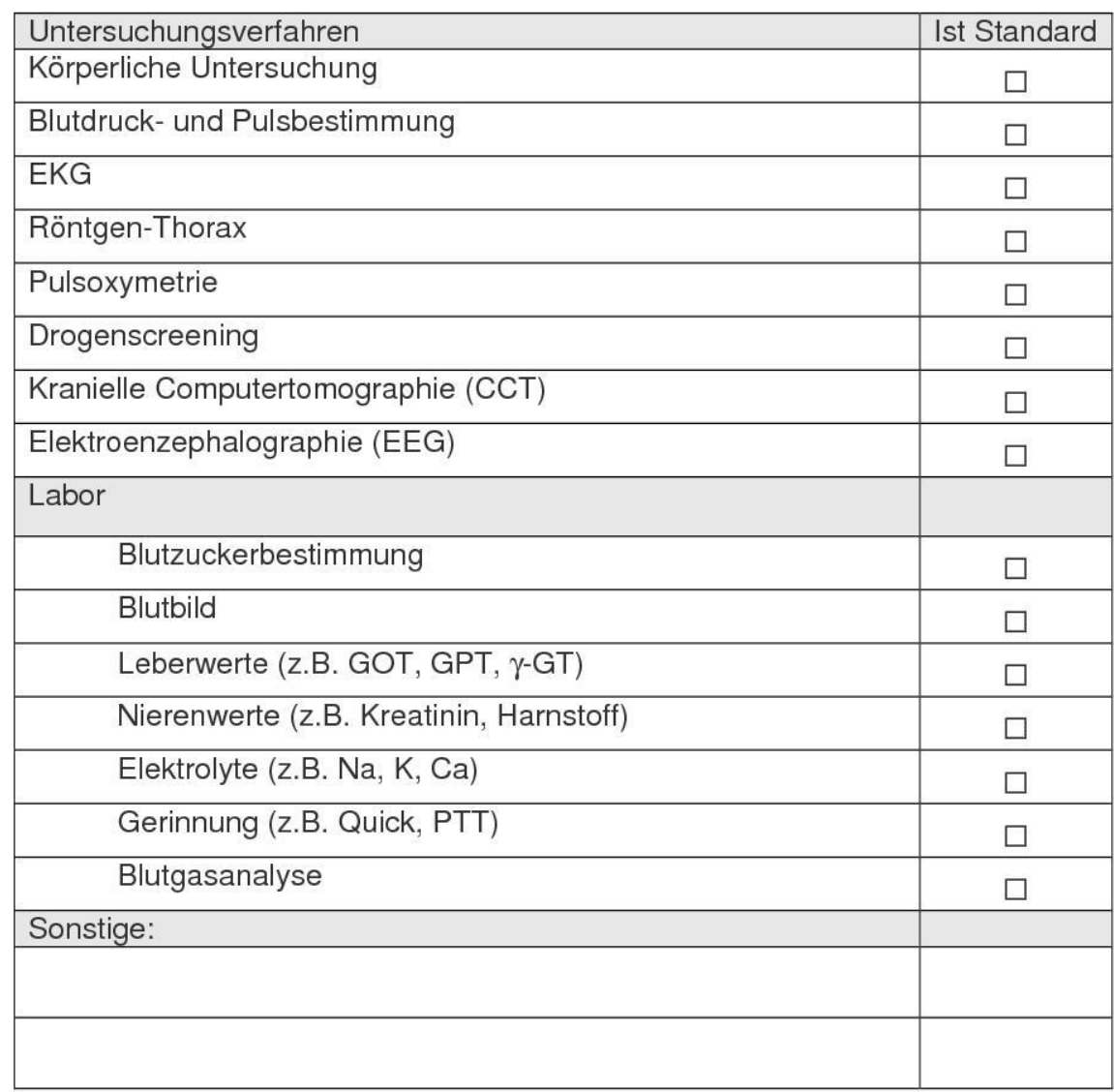


Welches der folgenden Medikamente, dass zur Behandlung psychiatrischer Störungen in der Notaufnahme geeignet sein könnte, ist in Ihrer Notaufnahme verfügbar?

\begin{tabular}{|c|c|c|}
\hline Präparat & oral & parenteral \\
\hline Lorazepam (z.B. Tavor ${ }^{(\mathbb{B})}$ & $\square$ & $\square$ \\
\hline Diazepam (z.B. Valium ${ }^{(8)}$ & $\square$ & $\square$ \\
\hline Promethazin (z.B. Atosil ${ }^{(\mathbb{})}$ ) & $\square$ & $\square$ \\
\hline Levomepromazin (z.B. Neurocil $\left.{ }^{(\theta)}\right)$ & $\square$ & $\square$ \\
\hline Haloperidol (z.B. Haldol ${ }^{\circledR}$ ) & $\square$ & $\square$ \\
\hline Amitryptilin (z.B. Saroten ret. ${ }^{\circledR}$, Amineurin $^{(\otimes)}$ ) & $\square$ & $\square$ \\
\hline Oxazepam (z.B. Adumbran ${ }^{(8)}$ ) & $\square$ & $\square$ \\
\hline Melperon (z.B. Eunerpan ${ }^{(8)}$ ) & $\square$ & $\square$ \\
\hline Pipamperon (z.B. Dipiperon ${ }^{(8)}$ ) & $\square$ & $\square$ \\
\hline \multicolumn{3}{|l|}{ weitere: } \\
\hline & $\square$ & $\square$ \\
\hline & $\square$ & $\square$ \\
\hline & $\square$ & $\square$ \\
\hline
\end{tabular}

Welches Medikament halten Sie für unverzichtbar in der Notaufnahme?

\begin{tabular}{|c|c|c|}
\hline Präparat & oral unverzichtbar & $\begin{array}{c}\text { parenteral } \\
\text { unverzichtbar }\end{array}$ \\
\hline Lorazepam (z.B. Tavor ${ }^{(\mathbb{Q})}$ & $\square$ & $\square$ \\
\hline Diazepam (z.B. Valium ${ }^{(2)}$ & $\square$ & $\square$ \\
\hline Promethazin (z.B. Atosi $\left.{ }^{(\theta)}\right)$ & $\square$ & $\square$ \\
\hline Levomepromazin (z.B. Neurocil $\left.{ }^{(\otimes)}\right)$ & $\square$ & $\square$ \\
\hline Haloperidol (z.B. Haldol $\left.{ }^{(\otimes)}\right)$ & $\square$ & $\square$ \\
\hline Amitryptilin (z.B. Saroten ret. ${ }^{\circledR}$, Amineurin $\left.^{(}\right)$ & $\square$ & $\square$ \\
\hline Oxazepam (z.B. Adumbran ${ }^{(ब)}$ ) & $\square$ & $\square$ \\
\hline Melperon (z.B. Eunerpan ${ }^{(2)}$ ) & $\square$ & $\square$ \\
\hline Pipamperon (z.B. Dipiperon ${ }^{(\mathbb{Q})}$ & $\square$ & $\square$ \\
\hline \multicolumn{3}{|l|}{ weitere: } \\
\hline & $\square$ & $\square$ \\
\hline & $\square$ & $\square$ \\
\hline & $\square$ & $\square$ \\
\hline
\end{tabular}


Welches weitere Medikament wünschen Sie sich in der Notaufnahme bzw. für welche Indikationen oder Situationen wünschen Sie sich weitere Medikamente? 
Wie würden in Ihrer Notaufnahme typischerweise folgende Notfallsituationen versorgt?

- Somnolent bis komatöser 28-jähriger mit schwerer, akuter Alkoholintoxikation $(2,1 \% \circ)$, ohne größere Störung der Vitalparameter

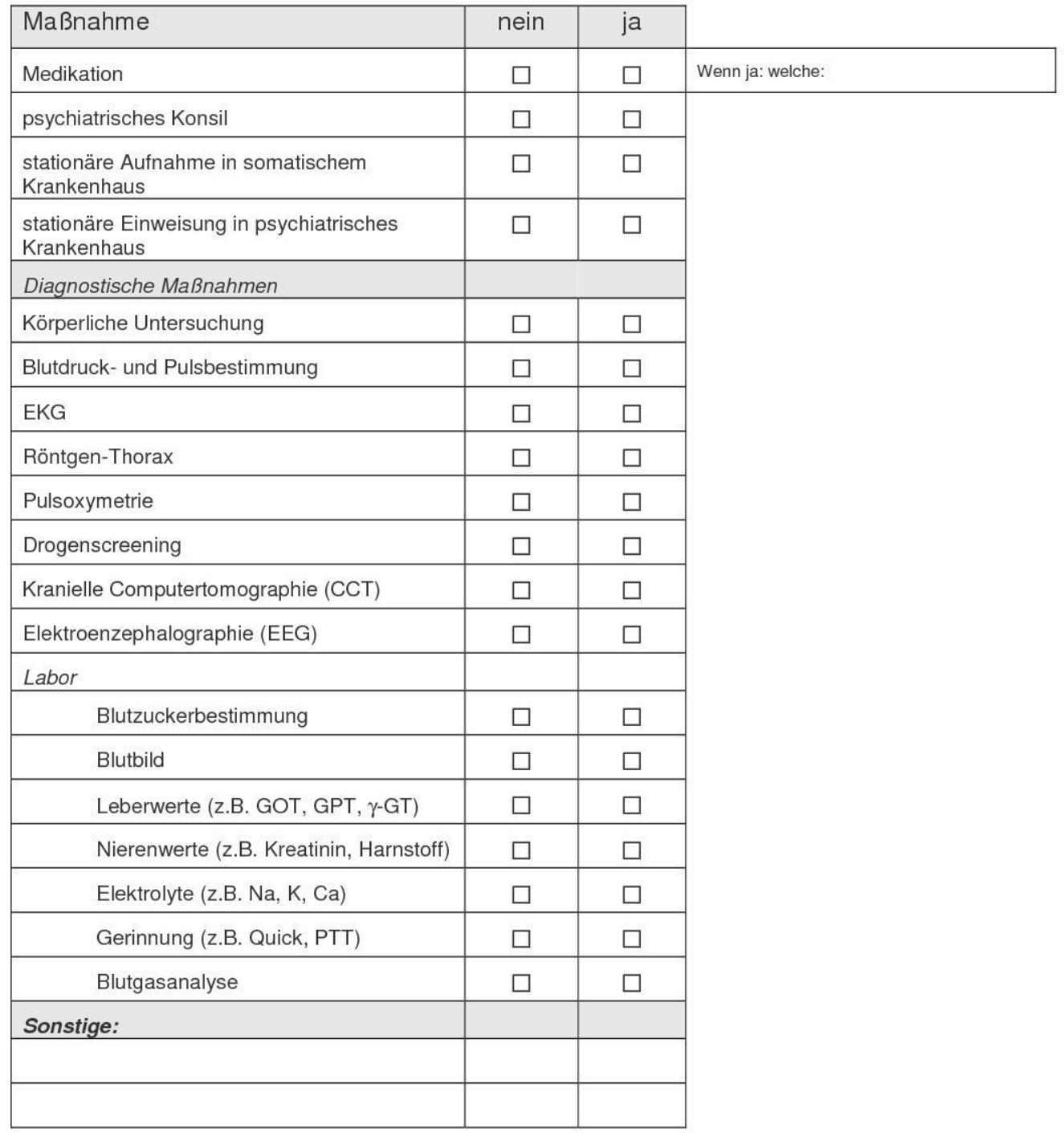


- akuter Verwirrtheitszustand bei einem multimorbiden, hochbetagten Patienten

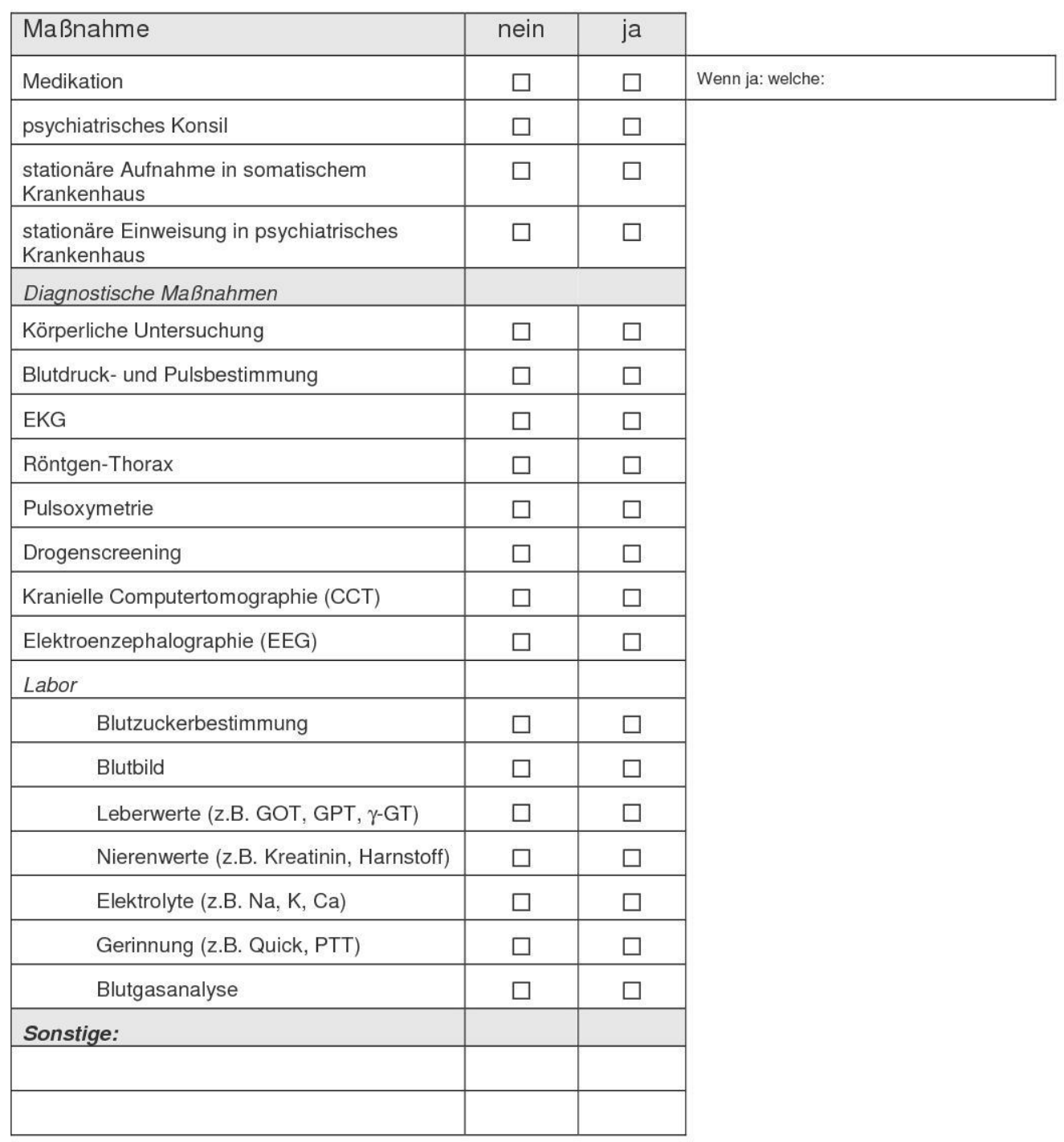


- Alkoholentzugsdelir eines typischen 48-jährigen Alkoholabhängigen

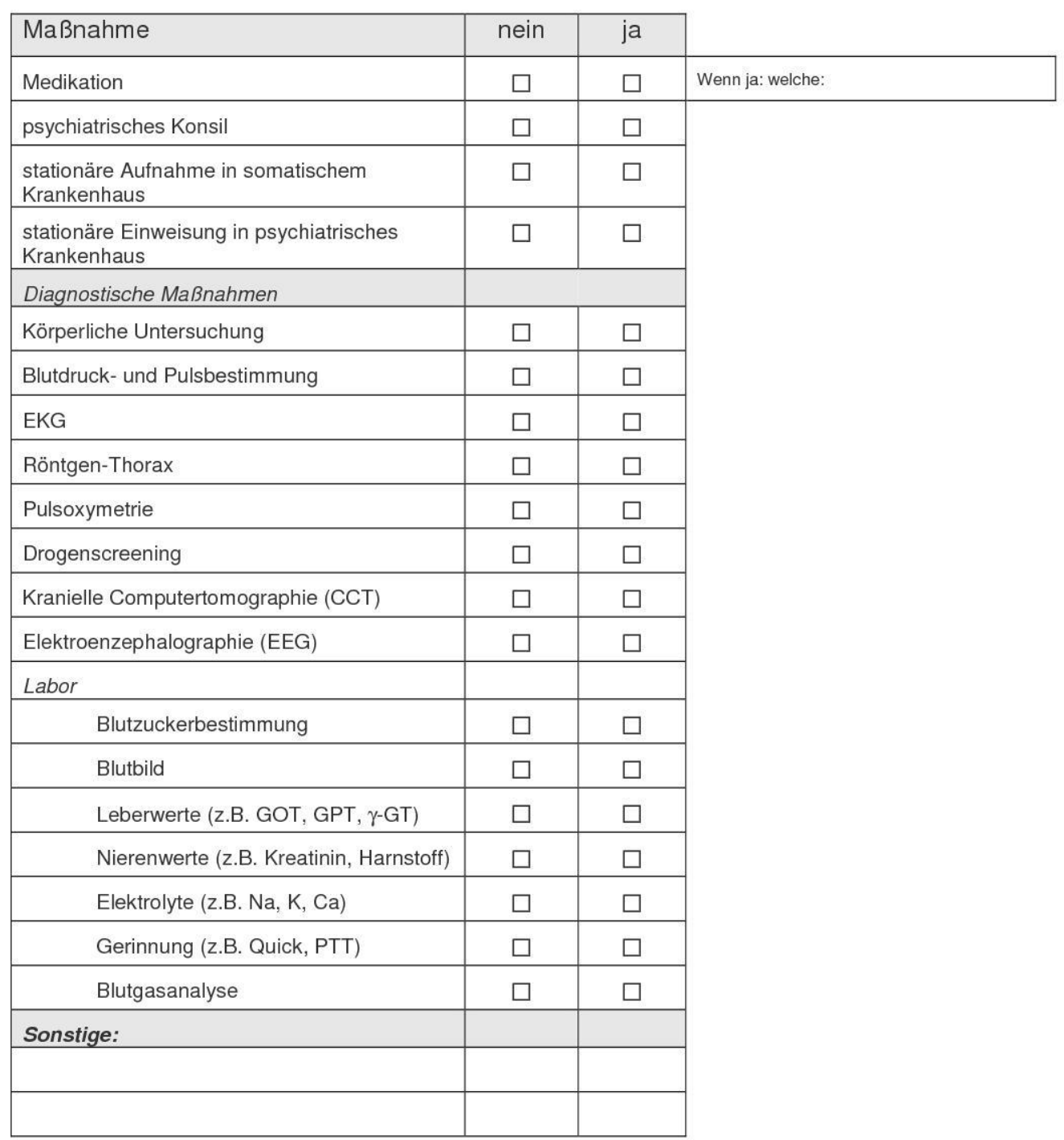


- Suizidversuch einer 32-jährigen Frau durch Einnahme von 10 Tbl. Aspirin und $10 \mathrm{Tbl}$. Adumbran nach Partnerschaftskonflikt, stark somnolent

\begin{tabular}{|c|c|c|c|}
\hline Maßnahme & nein & ja & \\
\hline Medikation & $\square$ & $\square$ & Wenn ja: welch \\
\hline psychiatrisches Konsil & $\square$ & $\square$ & \\
\hline $\begin{array}{l}\text { stationäre Aufnahme in somatischem } \\
\text { Krankenhaus }\end{array}$ & $\square$ & $\square$ & \\
\hline $\begin{array}{l}\text { stationäre Einweisung in psychiatrisches } \\
\text { Krankenhaus }\end{array}$ & $\square$ & $\square$ & \\
\hline \multicolumn{3}{|l|}{ Diagnostische Maßnahmen } & \\
\hline Körperliche Untersuchung & $\square$ & $\square$ & \\
\hline Blutdruck- und Pulsbestimmung & $\square$ & $\square$ & \\
\hline EKG & $\square$ & $\square$ & \\
\hline Röntgen-Thorax & $\square$ & $\square$ & \\
\hline Pulsoxymetrie & $\square$ & $\square$ & \\
\hline Drogenscreening & $\square$ & $\square$ & \\
\hline Kranielle Computertomographie (CCT) & $\square$ & $\square$ & \\
\hline Elektroenzephalographie (EEG) & $\square$ & $\square$ & \\
\hline \multicolumn{3}{|l|}{ Labor } & \\
\hline Blutzuckerbestimmung & $\square$ & $\square$ & \\
\hline Blutbild & $\square$ & $\square$ & \\
\hline Leberwerte (z.B. GOT, GPT, $\gamma$-GT) & $\square$ & $\square$ & \\
\hline Nierenwerte (z.B. Kreatinin, Harnstoff) & $\square$ & $\square$ & \\
\hline Elektrolyte (z.B. Na, K, Ca) & $\square$ & $\square$ & \\
\hline Gerinnung (z.B. Quick, PTT) & $\square$ & $\square$ & \\
\hline Blutgasanalyse & $\square$ & $\square$ & \\
\hline \multicolumn{3}{|l|}{ Sonstige: } & \\
\hline
\end{tabular}


- stuporöser 62-jähriger Patient ohne erkennbare organische Ursache

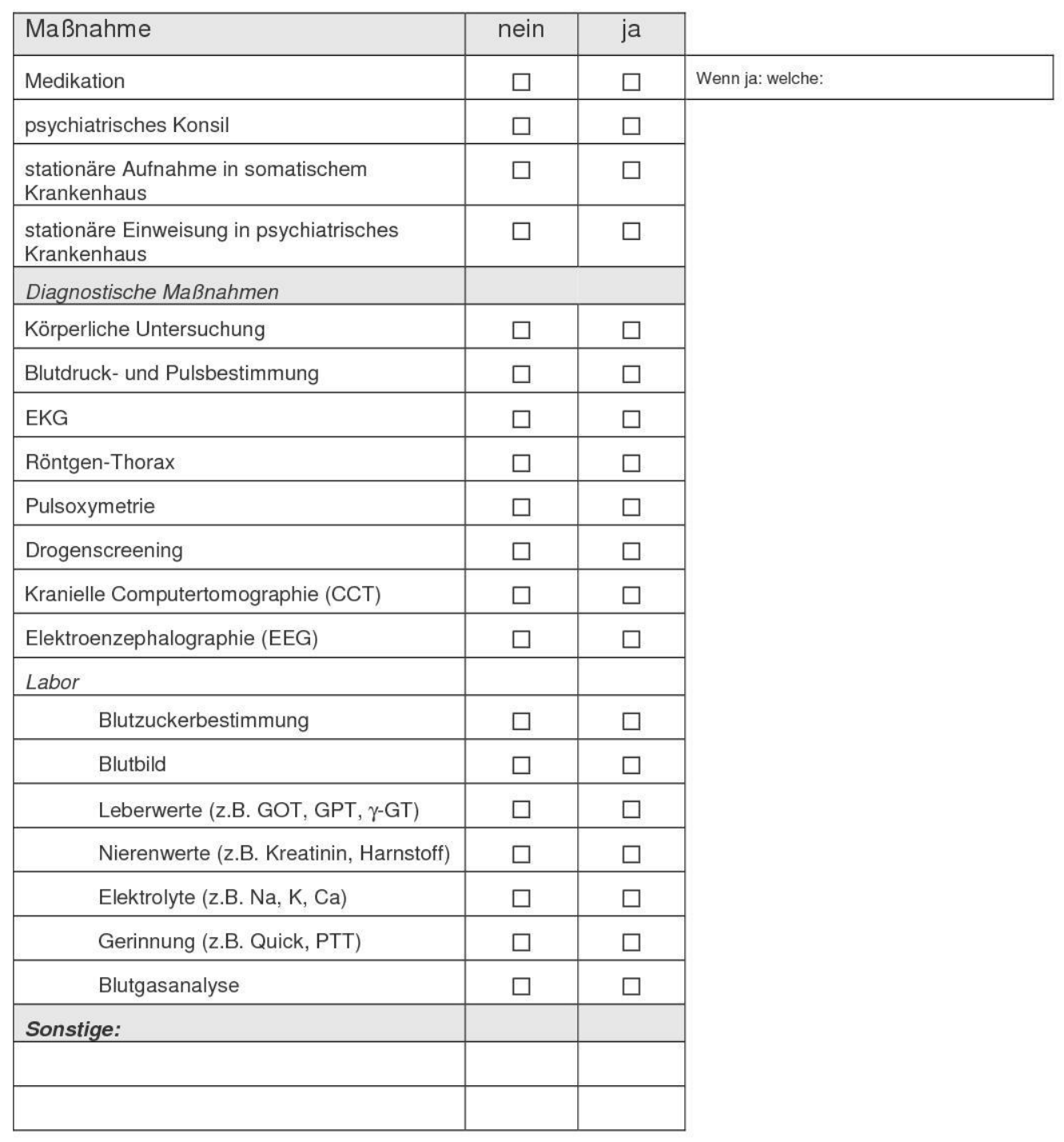


- 29-jährige schizophrene Patientin mit wieder aufgetretenen Halluzinationen und Verfolgungswahn nach unregelmäßiger Einnahme der Medikation

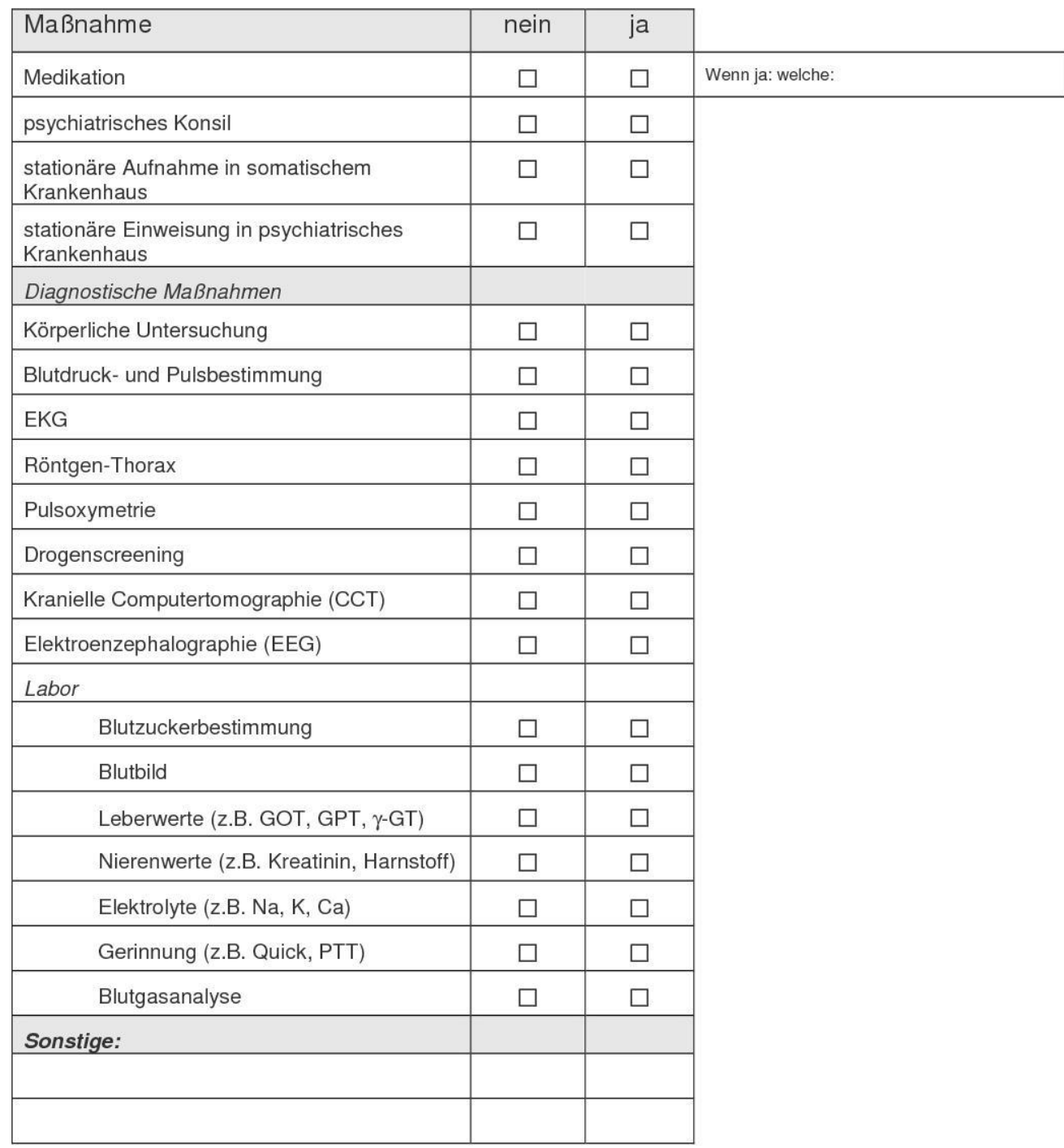

Vielen Dank für Ihre Mitarbeit und Zeit!

Wenn Sie eine Auswertung der Untersuchung wünschen, bitten wir Sie, eine email an folgende Adresse zu senden:

agpun@klinik-dr-fontheim.de 


\section{Publikationen/Dank}

\subsection{Publikationen}

Die Ergebnisse dieser Arbeit wurden in Auszügen bereits wie folgt veröffentlicht:

„Psychiatrische Notfallpatienten in allgemeinen Notaufnahmen“, Jahrestagung der Deutschen Gesellschaft für Psychiatrie, Psychotherapie und Nervenheilkunde (DGPPN), Berlin, 24.11. - 27.11.2010.

„Psychiatrische Notfallpatienten in allgemeinen Notaufnahmen“, 10.Kongress der Deutschen Interdisziplinären Vereinigung für Intensiv- und Notfallmedizin (DIVI), Hamburg, 01.12. - 04.12.2010.

„Psychiatrische Notfallpatienten in allgemeinen Notaufnahmen“, Deutscher Interdisziplinärer Notfallmedizin Kongress (DINK), Wiesbaden, 24.02. - 26.02.2011.

Ein Manuskript mit dem Titel „Psychiatrische Versorgung in der Notaufnahme“ ist fertiggestellt und wird bei der Zeitschrift „Der Anästhesist“ eingereicht.

\subsection{Danksagungen}

Herrn Prof. Dr. med. Frank-Gerald Pajonk danke ich für die Überlassung des Themas, die Hilfsbereitschaft und die sehr gute Betreuung bei der Durchführung der vorliegenden Arbeit.

Frau Beatrice Hessing und Herrn Carsten Zimmer danke ich für die Unterstützung und Motivation während der gesamten Arbeit.

Frau Kathrin Nolden und Herrn Julian Puffer danke ich für die kritische Durchsicht der vorliegenden Arbeit.

Zum Schluss bedanke ich mich bei allen Kolleginnen und Kollegen, die freundlicherweise die Fragebögen ausgefüllt haben. 


\section{Lebenslauf}

\section{PERSÖNLICHE DATEN}

Geburtsdatum/-ort:

26. April 1966 in Viersen

Nationalität:

deutsch

Familienstand:

ledig, keine Kinder

SCHULBILDUNG

1972 - 1976 katholische Grundschule

Sankt Marien, Viersen-Hamm

1976 - 1985 humanistisches Gymnasium,

Viersen; Abschluss mit der Allgemeinen

Hochschulreife

ZIVILDIENST

07/1985 - 02/1987 Städtisches Altenheim, Mönchengladbach-Lürrip

\section{BERUFLICHE BILDUNG}

Studium:
Ab 10/1987 Studium der Humanmedizin an der Universität - Gesamthochschule Essen
09/1990 Physikum
09/1991 Erstes Staatsexamen
09/1994 Zweites Staatsexamen
10/1995 Drittes Staatsexamen

ärztliche Tätigkeiten:

12/1995 - 11/1996 Arzt im Praktikum in der urologischen Abteilung des Marienhospitals Altenessen in Essen 12/1996 - 05/1997 Arzt im Praktikum im Städtischen Krankenhaus DresdenFriedrichstadt, Institut für Pathologie 
01/1998 - 03/1998 Assistenzarzt im Städtischen Klinikum Dortmund, Institut für

Pathologie

04/1998 - 06/1998 Assistenzarzt in den

Städtischen Krankenanstalten Bielefeld-

Mitte, Institut für Pathologie

Seit 07/1998 Assistenzarzt im Städtischen

Klinikum Dresden-Friedrichstadt, Institut für Pathologie

12/2002 Facharztanerkennung für Pathologie 